\title{
On the transfer between the Dickson algebras as modules over the Steenrod algebra
}

\author{
Võ T. N. Quỳnh • Lưu X. Trường
}

Received: 25 June 2014 / Accepted: 8 November 2014 / Published online: 11 December 2014 (C) Tbilisi Centre for Mathematical Sciences 2014

\begin{abstract}
Let $D_{\ell}:=D(\mathbb{W})$ be the Dickson-Mùi algebra of $\mathbb{W}$ for an elementary abelian $p$-group $\mathbb{W}$ of rank $\ell$, which consists of all invariants in the mod $p$ cohomology of $\mathbb{W}$ under the general linear group $G L(\mathbb{W})$. Hưng (Math Ann 353:827-866, 2012) determined explicitly all the homomorphisms between the Dickson-Mùi algebras (regarded as modules over the Steenrod algebra, $\mathcal{A}$ ). He showed that the compositions of the restrictions res $_{\ell, m}: D_{\ell} \rightarrow D_{m}$ and the transfers $t r_{m, n}: D_{m} \rightarrow D_{n}$ for $m \leq \min \{\ell, n\}$ form a basis of $\operatorname{Hom}_{\mathcal{A}}\left(D_{\ell}, D_{n}\right)$. The restriction res $_{\ell, m}$ has explicitly been known (from Lemmas 3.3 and 3.4 for $p=2$ and Lemma 9.1 for $p>2$ of the cited article), while the transfer $t r_{m, n}$ has only been computed for $m=1$ in some degrees (see Lemma 9.2 of the article). In this paper, we study $t r_{m, n}$ for $p=2$ in general. We determine completely $t r_{1, n}$ for any $n$, and compute the image of $t r_{m, n}$ for arbitrary $m, n$ on some powers of multilinear and alternating invariants. Then, we recognize some families of invariants in $D_{m}$ on which the transfer $t r_{m, n}$ vanishes.
\end{abstract}

Keywords Steenrod algebra · Modular representations · Invariant theory · Dickson algebra

Communicated by Lionel Schwartz.

Dedicated to Professor Nguyễn H. V. Hưng on the occasion of his 60th birthday.

This research is funded by Vietnam National Foundation for Science and Technology Development (NAFOSTED) under grant number 101.04 - 2014.19.

V. T. N. Quỳnh $(\bowtie) \cdot$ L. X. Trường

Department of Mathematics, Vietnam National University, Hanoi,

334 Nguyễn Trãi Street, Hanoi, Vietnam

e-mail: quynhvtn@vnu.edu.vn

L. X. Trường

e-mail: 1xtruong.1t@gmail.com 
Mathematics Subject Classification Primary 55S10 $55 \mathrm{~S} 05 \cdot 20 \mathrm{G} 10 \cdot 20 \mathrm{G} 05$

\section{Introduction}

Let $\mathbb{V}$ be an elementary abelian 2-group rank $n$. Then $\mathbb{V}$ can also be regarded as an $n$-dimensional vector space over $\mathbb{F}_{2}$, the field of two elements. Let $H^{*}(\mathbb{V})$ denote the cohomology of the group $\mathbb{V}$. As it is well-known, $H^{*}(\mathbb{V}) \cong S\left(\mathbb{V}^{*}\right)$ where $S\left(\mathbb{V}^{*}\right)$ denote the symmetric algebra over the space $\mathbb{V}^{*}$. Throughout the paper, the coefficient ring for homology and cohomology is always $\mathbb{F}_{2}$. Let $x_{1}, \ldots, x_{n}$ be a basis of $\mathbb{V}^{*}$, we have

$$
H^{*}(\mathbb{V}) \cong \mathbb{F}_{2}\left[x_{1}, \ldots, x_{n}\right] .
$$

The general linear group $G L(\mathbb{V}) \cong G L\left(n, \mathbb{F}_{2}\right)$ acts regularly on $\mathbb{V}$ and therefore on $H^{*}(\mathbb{V})$. The Dickson algebra is the algebra of all invariants of $H^{*}(\mathbb{V})$ under the action of $G L(\mathbb{V})$. It is explicitly determined by Dickson in [2] as follows:

$$
D(\mathbb{V}):=H^{*}(\mathbb{V})^{G L(\mathbb{V})} \cong \mathbb{F}_{2}\left[x_{1}, \ldots, x_{n}\right]^{G L\left(n, \mathbb{F}_{2}\right)}=\mathbb{F}_{2}\left[Q_{n, 0}, Q_{n, 1}, \ldots, Q_{n, n-1}\right],
$$

where $Q_{n, i}$ denotes the Dickson invariant of degree $2^{n}-2^{i}$. (A precise definition for the Dickson invariants will be given in Sect. 2.)

Being the cohomology of the classifying space $B \mathbb{V}$, the group $H^{*}(\mathbb{V})$ is equipped with a structure of module over the $\bmod 2$ Steenrod algebra, $\mathcal{A}$. Each $\gamma \in G L(\mathbb{V})$ induces an $\mathcal{A}$-isomorphism $\gamma^{*}$ on $H^{*}(\mathbb{V})$. The map $\gamma \mapsto \gamma^{*}$ gives rise to the regular action of $G L(\mathbb{V})$ on $H^{*}(\mathbb{V})$. So, the actions of $G L(\mathbb{V})$ and $\mathcal{A}$ on $H^{*}(\mathbb{V})$ commute with each other. Hence, the Dickson algebra $D(\mathbb{V})$ inherits a structure of module over the Steenrod algebra $\mathcal{A}$ from $H^{*}(\mathbb{V})$.

Let $\mathbb{U}$ be a $\mathbb{F}_{2}$-vector space of dimension $m$ with $m \leq n$. The subject of the present paper is the transfer $t r_{\mathbb{U}, \mathbb{V}}: D(\mathbb{U}) \rightarrow D(\mathbb{V})$, which is also denoted by $t r_{m, n}: D_{m} \rightarrow$ $D_{n}$ defined in [3]. For fixed $n$ and $\ell$, the set $\left\{t r_{m, n} \mid m<\min \{\ell, n\}\right\}$ is known as a key component forming all $\mathcal{A}$-homomorphisms from $D_{\ell}$ to $D_{n}$ in the sense as follows. Let $\mathbb{W}$ be a vector space of dimension $\ell$. The restriction res $\ell, m: D_{\ell} \rightarrow$ $D_{m}$ is the homomorphism induced from an inclusion $\mathbb{U} \rightarrow \mathbb{W}$ (in [3, Lemma 3.1], Hưng showed that any inclusion $\mathbb{U} \rightarrow \mathbb{W}$ induces the same homomorphism on $\left.H^{*}(\mathbb{W})^{G K(\mathbb{W})}\right)$. Let $\bar{D}(\mathbb{V})$ or $\bar{D}_{n}$ be the augmentation ideal of all positive degree elements in the Dickson algebra $D_{n}$.

Theorem 1.1 [3, Theorem 1.1] The A-module homomorphisms $\left\{\operatorname{tr}_{m, n}\right.$ res $_{\ell, m}: 1 \leq$ $m \leq \min \{\ell, n\}\}$ form a basis of the vector space $\operatorname{Hom}_{\mathcal{A}}\left(\bar{D}_{\ell}, \bar{D}_{n}\right)$ of $\mathcal{A}$-module homomorphisms from $\bar{D}_{\ell}$ to $\bar{D}_{n}$. In particular, $\operatorname{dim} \operatorname{Hom}_{\mathcal{A}}\left(\bar{D}_{\ell}, \bar{D}_{n}\right)=\min \{\ell, n\}$.

The restrictions res $_{\ell, m}$ are explicitly determined on each Dickson monomial in [3, Lemma 3.4]; so, in order to compute $\operatorname{Hom}_{\mathcal{A}}\left(\bar{D}_{\ell}, \bar{D}_{n}\right)$ we need to compute the transfers $t r_{m, n}$.

First, the transfer $\operatorname{tr}_{1, n}$ is concretely computed in [3, Lemma 4.2]. Then, it is further studied in the undergraduate thesis of Phạm H. Đăng under the guidance of Nguyễn H. V. Hưng (see [1]).

In the case $m=1$, we have $D_{m}=\mathbb{F}_{2}[x]$ in which $\operatorname{deg} x=1$. The following theorem is one of main results of the paper. 
Theorem 1.2 For $k>0$,

$$
\operatorname{tr}_{1, n}\left(x^{k}\right)=\sum_{\sum_{i=0}^{n-1}\left(2^{n}-2^{i}\right) s_{i}=k} \frac{\left(\sum_{i=0}^{n-1} s_{i}-1\right) !}{\left(s_{0}-1\right) ! s_{1} ! \ldots s_{n-1} !} Q_{n, 0}^{s_{0}} Q_{n, 1}^{s_{1}} \ldots Q_{n, n-1}^{s_{n-1}} .
$$

Here $\frac{\left(\sum_{i=0}^{n-1} s_{i}-1\right) !}{\left(s_{0}-1\right) ! s_{1} ! \ldots s_{n-1} !}=0$ if $s_{0}=0$.

Let $x_{1}, \ldots, x_{m}$ be a basis for $\mathbb{U}^{*}$. Then each $f \in D_{m}=D(\mathbb{U})$ is a polynomial in the variables $x_{i}(1 \leq i \leq m)$; so, we rewrite $f$ as $f\left(x_{1}, \ldots, x_{m}\right)$. We denote by $f$ the map $\underbrace{\mathbb{V}^{*} \times \cdots \times \mathbb{V}^{*}} \rightarrow S\left(\mathbb{V}^{*}\right),\left(t_{1}, \ldots, t_{m}\right) \mapsto f\left(t_{1}, \ldots, t_{m}\right)$. We say that $f\left(x_{1}, \ldots, x_{m}\right)$ is multilinear on $\mathbb{V}^{*}$ if $f$ is $m$-multilinear. The invariant $f\left(x_{1}, \ldots, x_{m}\right)$ is called alternating on $\mathbb{V}^{*}$ if the map $f$ is alternating.

The set of invariants in $D_{m}$ which are multilinear and alternating forms a subspace of $D_{m}$. Furthermore, this subspace is subset of the ideal of $D_{m}$ generated by $Q_{m, 0}$ (see Corollary 4.7). It is infinity dimensional and can be explicitly determined as follows. For each set of $m$ distinct non-negative numbers $\beta=\left\{\beta_{1}, \ldots, \beta_{m}\right\}$, let $\Sigma_{m}$ be the symmetric group on $\{1, \ldots, m\}$ and let

$$
\omega_{\beta}\left(x_{1}, \ldots, x_{m}\right)=\sum_{\sigma \in \Sigma_{m}} x_{1}^{2^{\beta_{\sigma(1)}}} \ldots x_{m}^{2^{\beta_{\sigma(m)}}} .
$$

Proposition 1.3 The set

$$
\left\{\omega_{\beta}\left(x_{1}, \ldots, x_{m}\right) \mid \beta=\left(\beta_{1}, \ldots, \beta_{m}\right) \in \mathbb{N}^{m}, \quad \beta_{i} \neq \beta_{j} \text { for all } i \neq j\right\}
$$

forms a basis for the subspace of $D_{m}$ generated by multilinear and alternating invariants.

The following theorem shows the image of the transfer $t r_{m, m+1}$ on powers of multilinear and alternating invariants in $D_{m}$. For each $Q \in D_{m}$ and $y_{1}, \ldots, y_{m} \in$ $\mathbb{F}\left[x_{1}, \ldots, x_{m+1}\right]$, we denote by $Q\left(y_{1}, \ldots, y_{m}\right)$ the polynomial in $\mathbb{F}\left[x_{1}, \ldots, x_{m+1}\right]$ obtained from $Q$ by the substitution $y_{j}$ for $x_{j}(j=1, \ldots, m)$.

Theorem 1.4 Suppose that $Q$ is a multilinear and alternating invariant in $D_{m}$. Let $y_{j}=Q\left(x_{1}, \ldots, x_{j-1}, x_{j+1}, \ldots, x_{m+1}\right)$ for $j=1, \ldots, m+1$. Then, for $r>0$ we have

$$
t r_{m, m+1}\left(Q^{r}\right)=\sum \frac{\left(s_{0}+\cdots+s_{m}-1\right) !}{\left(s_{0}-1\right) ! s_{1} ! \ldots s_{m} !}\left(Q_{m+1,0}^{s_{0}} \ldots Q_{m+1, m}^{s_{m}}\right)\left(y_{1}, \ldots, y_{m+1}\right)
$$

summed over all sequences of non-negative integers $\left(s_{0}, \ldots, s_{m}\right)$ such that

$$
\sum_{i=0}^{m}\left(2^{m+1}-2^{i}\right) s_{i}=r
$$


Since $Q_{m, 0}, Q_{m, 0} Q_{m, i}$ are multilinear and alternating invariants (see Example 4.8), we get the two following explicit formulas for image of $t r_{m, m+1}$ on some powers of these invariants.

Corollary 1.5 (i) $t r_{m, m+1}\left(Q_{m, 0}^{j}\right)=0$ for $0<j<2^{m+1}-1$.

(ii) $t r_{m, m+1}\left(Q_{m, 0}^{2^{m+1}-1}\right)=Q_{m+1,0}^{2^{m}-1}$.

Corollary 1.6 For $r>0, \operatorname{tr}_{2,3}\left(Q_{2,0}^{r} Q_{2,1}^{r}\right)$

$$
\begin{aligned}
= & \sum_{7 s_{0}+6 s_{1}+4 s_{2}=r} \frac{\left(s_{0}+s_{1}+s_{2}-1\right) !}{\left(s_{0}-1\right) ! s_{1} ! s_{2} !}\left(Q_{3,0}^{3} Q_{3,1} Q_{3,2}^{2}+Q_{3,0}^{5}\right)^{s_{0}}\left(Q_{3,0}^{2} Q_{3,2}^{4}\right)^{s_{1}} \\
& \times\left(Q_{3,1}^{2} Q_{3,2}^{2}+Q_{3,0}^{2} Q_{3,1}\right)^{s_{2}} .
\end{aligned}
$$

The following proposition gives some families of invariants on which the transfer is zero.

Proposition $1.7 \operatorname{tr}_{m, n}(Q)$ is zero for each of the following cases.

(i) $Q \in D_{m}$ is a multilinear and alternating invariant;

(ii) $Q=Q_{m, 0}^{2^{s_{0}}} Q_{m, 1}^{2^{s_{1}}} \ldots Q_{m, m-1}^{2^{s_{m-1}}}$ where $s_{0}, s_{1}, \ldots, s_{m-1}$ are nonnegative with $m, n$ such that $n \geq \frac{3 m^{2}+3 m}{2}$;

(iii) $m=2, n \geq 4$ and $Q=Q_{2,0}^{2^{s_{0}}} Q_{2,1}^{2^{s_{1}}}$ for any nonnegative integers $s_{0}, s_{1}$.

The statement in Proposition 1.7(iii) is no longer true for the case $n=3$. In fact, $\operatorname{tr}_{2,3}\left(Q_{2,0}^{2^{s_{0}}} Q_{2,1}^{2^{s_{1}}}\right) \neq 0$ for $s_{0}-s_{1}>1$ or $s_{0}-s_{1}<0$ (see Remark 7.3).

The paper is divided into seven sections. The introduction in Sect. 1 is followed by the preliminary in Sect. 2, where we recall definition of the transfer between the Dickson algebras. Section 3 is a proof of Theorem 1.2. The concepts of multilinear and alternating invariants are introduced in Sect. 4, where we prove Proposition 1.3. In Sect. 5, we study the transfer on powers of multilinear and alternating invariants, then prove Theorem 1.4 and Proposition 1.7(i). Section 6 deals with a method of finding invariant monomials in the image of the transfer by finding "leading elements" of it. By using this method, we prove Lemma 5.5, the most technical lemma of the paper that is used in the proofs of Corollaries 1.5 and 1.6. Finally, in Sect. 7, we prove Proposition 1.7(ii) and (iii).

The paper was in progress while the first named author was visiting to the Vietnam Institute for Advanced Study in Mathematics (VIASM). She would like to thank VIASM for the financial support and the warm hospitality.

\section{Preliminary}

In this section, we exploit Hưng's definition of the transfer $t r_{m, n}$.

Let $\mathbb{U}$ and $\mathbb{V}$ be a $\mathbb{F}_{2}$-vector spaces of dimensions $m$ and $n$ with $m \leq n$. Let $\mathbb{K}$ be a subspace of $\mathbb{V}$ and $\pi_{\mathbb{K}}: \mathbb{V} \rightarrow \mathbb{U}$ be an epimorphism with ker $\pi_{\mathbb{K}}=K$. Suppose that $\pi: \mathbb{V} \rightarrow \mathbb{U}$ is another epimorphism whose kernel is also $\mathbb{K}$. Then 
there is an isomorphism $\alpha: \mathbb{U} \rightarrow \mathbb{U}$ such that $\pi_{\mathbb{K}}=\alpha \pi$. It follows that $\pi_{\mathbb{K}}^{*}=$ $\pi^{*} \alpha^{*}: H^{*}(\mathbb{U}) \rightarrow H^{*}(\mathbb{V})$. Since $\alpha^{*}$ acts identically on the invariants $H^{*}(\mathbb{U})^{G L(\mathbb{U})}$, $\pi_{\mathbb{K}}^{*}$ and $\pi^{*}$ are the same on $H^{*}(\mathbb{U})^{G L(\mathbb{U})}$. Therefore, the induced homomorphism $\pi_{\mathbb{K}}^{*}: H^{*}(\mathbb{U})^{G L(\mathbb{U})} \rightarrow H^{*}(\mathbb{V})$ does not depend on the choice of the epimorphism $\pi_{K}$. It only depends on the kernel of $\pi_{\mathbb{K}}, \mathbb{K}$. On the other hand, the group $G L(\mathbb{V})$ permute the subspaces $\mathbb{K}$ of dimension $n-m$ in $\mathbb{V}$. So the sum of $\pi_{\mathbb{K}}^{*}$ maps $H^{*}(\mathbb{U})^{G L(\mathbb{U})}$ to the $G L(\mathbb{V})$-invariants.

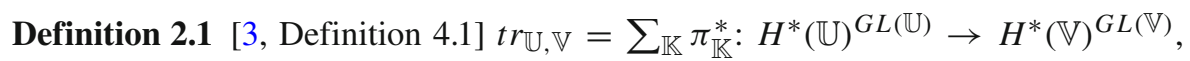
where the sum runs over all the subspaces $\mathbb{K}$ of dimension $(\operatorname{dim} \mathbb{V}-\operatorname{dim} \mathbb{U})$ in $\mathbb{V}$.

As it is well known that $H^{1}(\mathbb{V}) \cong \mathbb{V}^{*}$ and $H^{*}(\mathbb{V}) \cong S\left(\mathbb{V}^{*}\right)$ where $S\left(\mathbb{V}^{*}\right)$ denote the symmetric algebra over the space $\mathbb{V}^{*}$. Let $x_{1}, \ldots, x_{n}$ be a basis of $\mathbb{V}^{*}$, we have

$$
H^{*}(\mathbb{V}) \cong \mathbb{F}_{2}\left[x_{1}, \ldots, x_{n}\right]
$$

Recall that the algebra of the $G L(\mathbb{V})$-invariants $H^{*}(\mathbb{V})^{G L(\mathbb{V})}$ was computed by Dickson [2]. He showed in [2] that $H^{*}(\mathbb{V})^{G L(\mathbb{V})}$ is also a polynomial algebra and denoted it by $D_{n}$ :

$$
D_{n}:=H^{*}(\mathbb{V})^{G L(\mathbb{V})}=\mathbb{F}_{2}\left[Q_{n, 0}, Q_{n, 1}, \ldots, Q_{n, n-1}\right]
$$

Here $Q_{n, 0}, Q_{n, 1}, \ldots, Q_{n, n-1}$ are the Dickson invariants. They are inductively defined by the formula

$$
Q_{n, i}=Q_{n-1, i-1}^{2}+V_{n} Q_{n-1, i}
$$

where, by convention, $Q_{n, n}=1, Q_{n, i}=0$ for $i<0$, and

$$
V_{n}=\prod_{c_{j} \in \mathbb{F}_{2}}\left(c_{1} x_{1}+\cdots+c_{n-1} x_{n-1}+x_{n}\right)
$$

is the Mùi invariant under the Sylow 2-subgroup $T_{n}$ of $G L_{n}$ consisting of all upper triangular $n \times n$-matrices with 1 on the main diagonal (see Mùi [5]).

Since the transfer $t r_{\mathbb{U}, \mathbb{V}}$ only depends on the dimensions of $\mathbb{U}$ and $\mathbb{V}$, it is denoted by $t r_{m, n}: D_{m} \rightarrow D_{n}$, where $m=\operatorname{dim} \mathbb{U}$ and $n=\operatorname{dim} \mathbb{V}$. For each epimorphism $\pi: \mathbb{V} \rightarrow \mathbb{U}$, the induced homomorphism in cohomological degree $1, \pi^{*}: H^{1}(\mathbb{U}) \rightarrow$ $H^{1}(\mathbb{V})$ is a monomorphism. So $W:=\pi^{*} H^{1}(\mathbb{U})$ is a subspace of dimension $m$ in $H^{1}(\mathbb{V})$. Let $x_{1}, \ldots, x_{m}$ be a basis of $H^{1}(\mathbb{U})$. Then $\pi^{*}\left(x_{1}\right), \ldots, \pi^{*}\left(x_{m}\right)$ a basis for $\pi^{*} H^{1}(\mathbb{U})$. And for each $Q \in D_{m}$ we have $\pi^{*}(Q)=Q\left(\pi^{*}\left(x_{1}\right), \ldots, \pi^{*}\left(x_{m}\right)\right)$. Since $Q$ is invariant under any invertible transformation on $\mathbb{U}, Q\left(\pi^{*}\left(x_{1}\right), \ldots, \pi^{*}\left(x_{m}\right)\right)=$ $Q\left(y_{1}, \ldots, y_{m}\right)$ for any basis $y_{1}, \ldots, y_{m}$ of $W$. Hence $Q\left(\pi^{*}\left(x_{1}\right), \ldots, \pi^{*}\left(x_{m}\right)\right)$ only depends on $W$; so, we denote it by $Q(W)$ for short. Therefore, by Definition 2.1 we have

$$
t r_{m, n}(Q)=\sum_{W} Q(W)
$$


where the sum runs over all $m$-dimensional subspaces $W$ in $H^{1}(\mathbb{V})$. For example, with $m=1, n=3$ let $x_{1}, x_{2}, x_{3}$ be a basis for $\mathbb{V}^{*}$ we have

$\operatorname{tr}_{1,3}\left(x^{k}\right)=x_{1}^{k}+x_{2}^{k}+x_{3}^{k}+\left(x_{1}+x_{2}\right)^{k}+\left(x_{1}+x_{3}\right)^{k}+\left(x_{2}+x_{3}\right)^{k}+\left(x_{1}+x_{2}+x_{3}\right)^{k}$

since all one-dimensional subspaces of $\mathbb{V}^{*}$ are

$$
\left\langle x_{1}\right\rangle,\left\langle x_{2}\right\rangle,\left\langle x_{3}\right\rangle,\left\langle x_{1}+x_{2}\right\rangle,\left\langle x_{1}+x_{3}\right\rangle,\left\langle x_{2}+x_{3}\right\rangle,\left\langle x_{1}+x_{2}+x_{3}\right\rangle .
$$

\section{The transfer from $D_{1}$ to $D_{n}$}

The aim of this section is to prove the following theorem, which is also numbered as Theorem 1.2 in the introduction.

Theorem 3.1 For $k>0$,

$$
\operatorname{tr}_{1, n}\left(x^{k}\right)=\sum_{\sum_{i=0}^{n-1}\left(2^{n}-2^{i}\right) s_{i}=k} \frac{\left(\sum_{i=0}^{n-1} s_{i}-1\right) !}{\left(s_{0}-1\right) ! s_{1} ! \ldots s_{n-1} !} Q_{n, 0}^{s_{0}} Q_{n, 1}^{s_{1}} \ldots Q_{n, n-1}^{s_{n-1}} .
$$

Here $\frac{\left(\sum_{i=0}^{n-1} s_{i}-1\right) !}{\left(s_{0}-1\right) ! s_{1} ! \ldots s_{n-1} !}=0$ if $s_{0}=0$.

At small degrees, $\operatorname{tr}_{1, n}$ was explicitly determined by Hưng in [3] as follows.

Lemma 3.2 [3, Lemma 4.2]

(i) $\operatorname{tr}_{1, n}\left(x^{2^{n}-1}\right)=Q_{n, 0}$.

(ii) $\operatorname{tr}_{1, n}\left(x^{j}\right)$, for $0<j<2^{n}-1$.

In order to prove Theorem 3.1, we need Lemma 3.2 and the following inductive formula.

Lemma $3.3 \operatorname{tr}_{1, n}\left(x^{k}\right)=\sum_{i=0}^{n-1} Q_{n, i} \operatorname{tr}_{1, n}\left(x^{k-2^{n}+2^{i}}\right)$ for $k \geq 2^{n}-1$.

Proof The lemma is proved by induction on $n$. As $k \geq 2^{n}-1$, we rewrite $k$ as the form $k=\ell+2^{n}-1$ for $\ell \geq 0$. Then the lemma is equivalent to

$$
\operatorname{tr}_{1, n}\left(x^{\ell+2^{n}-1}\right)=\sum_{i=0}^{n-1} Q_{n, i} \operatorname{tr}_{1, n}\left(x^{\ell+2^{i}-1}\right)
$$

for $\ell \geq 0$.

For $n=1$, as $\operatorname{tr}_{1,1}$ is the identity map, we have $\operatorname{tr}_{1,1}\left(x^{\ell+1}\right)=x^{\ell+1}=$ $Q_{1,0} \operatorname{tr}_{1,1}\left(x^{\ell}\right)$. So the statement holds for $n=1$. Suppose inductively that it is true for $n$. Let

$$
\begin{aligned}
U & =\left\{c_{1} x_{1}+\cdots+c_{n} x_{n} \mid c_{i} \in \mathbb{F}_{2} \quad \text { for } i=1, \ldots, n\right\}, \\
W & =\left\{c_{1} x_{1}+\cdots+c_{n} x_{n}+x_{n+1} \mid c_{i} \in \mathbb{F}_{2} \quad \text { for } i=1, \ldots, n\right\} .
\end{aligned}
$$


For $s \geq 0$, we set

$$
R_{s}^{U}:=\sum_{x \in U} x^{s} \quad \text { and } \quad R_{s}^{W}:=\sum_{x \in W} x^{s}
$$

Then, by the definition of $t r_{1, .}$, we have $t r_{1, n}\left(x^{s}\right)=R_{s}^{U}$ and $t r_{1, n+1}\left(x^{s}\right)=R_{s}^{U}+R_{s}^{W}$. Furthermore, we get the two following formulas. They are proved latter.
(a) $\sum_{i=0}^{n-1} Q_{n, i}^{2} \operatorname{tr}_{1, n+1}\left(x^{\ell+2^{i+1}-1}\right)=R_{\ell+2^{n+1}-1}^{U}+\sum_{i=0}^{n-1} Q_{n, i}^{2} R_{\ell+2^{i+1}-1}^{W}$,
(b) $V_{n+1} \sum_{i=0}^{n} Q_{n, i}^{2} \operatorname{tr}_{1, n+1}\left(x^{\ell+2^{i}-1}\right)=R_{\ell+2^{n+1}-1}^{W}+\sum_{i=0}^{n-1} Q_{n, i}^{2} R_{\ell+2^{i+1}-1}^{W}$.

Then, we have

$$
\begin{aligned}
\sum_{i=0}^{n} Q_{n+1, i} \operatorname{tr}_{1, n+1}\left(x^{\ell+2^{i}-1}\right) & \\
= & \sum_{i=0}^{n}\left(Q_{n, i-1}^{2}+Q_{n, i} V_{n+1}\right) \operatorname{tr}_{1, n+1}\left(x^{\ell+2^{i}-1}\right) \\
& \left(\text { as } Q_{n+1, i}=Q_{n, i-1}^{2}+Q_{n, i} V_{n+1} \text { for } i \geq 0 \text { and } Q_{n, i}=0 \text { for } i<0\right) \\
= & \sum_{i=1}^{n} Q_{n, i-1}^{2} \operatorname{tr}_{1, n+1}\left(x^{\ell+2^{i}-1}\right)+V_{n+1} \sum_{i=0}^{n} Q_{n, i} \operatorname{tr}_{1, n+1}\left(x^{\ell+2^{i}-1}\right) \\
= & \sum_{i=0}^{n-1} Q_{n, i}^{2} \operatorname{tr}_{1, n+1}\left(x^{\ell+2^{i+1}-1}\right)+\left(R_{\ell+2^{n+1}-1}^{W}+\sum_{i=0}^{n-1} Q_{n, i}^{2} R_{\ell+2^{i+1}-1}^{W}\right) \\
& {[\operatorname{applying}(\mathrm{b})] } \\
= & R_{\ell+2^{n+1}-1}^{U}+R_{\ell+2^{n+1}-1}^{W} \quad[\text { applying (a)] } \\
= & \operatorname{tr}_{1, n+1}\left(x^{\ell+2^{n+1}-1}\right) .
\end{aligned}
$$

So, the lemma is proved.

Now, we prove the two formulas (a) and (b) above.

Case (a)

$$
\begin{aligned}
\operatorname{tr}_{1, n}\left(x^{\ell+2^{n+1}-1}\right) & =\operatorname{tr}_{1, n}\left(x^{\left(\ell+2^{n}\right)+2^{n}-1}\right) \\
& =\sum_{i=0}^{n-1} Q_{n, i} \operatorname{tr}_{1, n}\left(x^{\ell+2^{n}+2^{i}-1}\right)
\end{aligned}
$$

(by the inductive hypothesis on $t r_{1, n}$ for $\ell+2^{n}$ )

$$
=\sum_{i=0}^{n-1} \sum_{j=0}^{n-1} Q_{n, i} Q_{n, j} \operatorname{tr} r_{1, n}\left(x^{\left(\ell+2^{i}\right)+2^{j}-1}\right)
$$

[applying the inductive hypothesis to each $\operatorname{tr}_{1, n}\left(x^{\ell+2^{n}+2^{i}-1}\right)$ ]. 
However, we have

$$
\begin{aligned}
\sum_{i \neq j} Q_{n, i} Q_{n, j} \operatorname{tr}_{1, n}\left(x^{\left(\ell+2^{i}\right)+2^{j}-1}\right) & =\sum_{i<j}\left(Q_{n, i} Q_{n, j}+Q_{n, j} Q_{n, i}\right) \operatorname{tr}_{1, n}\left(x^{\ell+2^{i}+2^{j}-1}\right) \\
& =0 .
\end{aligned}
$$

Therefore

$$
\operatorname{tr}_{1, n}\left(x^{\ell+2^{n+1}-1}\right)=\sum_{i=0}^{n-1} Q_{n, i}^{2} \operatorname{tr}_{1, n}\left(x^{\left(\ell+2^{i+1}-1\right.}\right)
$$

It is equivalent to

$$
R_{x^{\ell+2^{n+1}-1}}^{U}=\sum_{i=0}^{n-1} Q_{n, i}^{2} \operatorname{tr}_{1, n}\left(x^{\left(\ell+2^{i+1}-1\right.}\right) .
$$

Then, we get

$$
\begin{aligned}
\sum_{i=0}^{n-1} Q_{n, i}^{2} t r_{1, n+1}\left(x^{\ell+2^{i+1}-1}\right) & =\sum_{i=0}^{n-1} Q_{n, i}^{2}\left(R_{\ell+2^{i+1}-1}^{U}+R_{\ell+2^{i+1}-1}^{W}\right) \\
& =\sum_{i=0}^{n-1} Q_{n, i}^{2} R_{\ell+2^{i+1}-1}^{U}+\sum_{i=0}^{n-1} Q_{n, i}^{2} R_{\ell+2^{i+1}-1}^{W} \\
& =R_{\ell+2^{n+1}-1}^{U}+\sum_{i=0}^{n-1} Q_{n, i}^{2} R_{\ell+2^{i+1}-1}^{W}
\end{aligned}
$$

So, we obtain (a).

Case (b). We have

$$
\sum_{i=0}^{n} Q_{n, i} \operatorname{tr}_{1, n+1}\left(x^{\ell+2^{i}-1}\right)=\sum_{i=0}^{n} Q_{n, i}\left(R_{\ell+2^{i}-1}^{U}+R_{\ell+2^{i}-1}^{W}\right) .
$$

Since the inductive hypothesis $\operatorname{tr}_{1, n}\left(x^{\ell+2^{n}-1}\right)=\sum_{i=0}^{n-1} Q_{n, i} t_{1, n}\left(x^{\ell+2^{i}-1}\right)$ and $Q_{n, n}=1$, it follows that

$$
\sum_{i=0}^{n} Q_{n, i} \operatorname{tr}_{1, n}\left(x^{\ell+2^{i}-1}\right)=0
$$

In the other word

$$
\sum_{i=0}^{n} Q_{n, i} R_{\ell+2^{i}-1}^{U}=0
$$


So

$$
\begin{aligned}
\sum_{i=0}^{n} Q_{n, i}^{2} \operatorname{tr}_{1, n+1}\left(x^{\ell+2^{i}-1}\right) & =\sum_{i=0}^{n} Q_{n, i}^{2}\left(R_{\ell+2^{i}-1}^{U}+R_{\ell+2^{i}-1}^{W}\right) \\
& =\sum_{i=0}^{n} Q_{n, i}^{2} R_{\ell+2^{i}-1}^{U}+\sum_{i=0}^{n} Q_{n, i}^{2} R_{\ell+2^{i}-1}^{W} \\
& =\sum_{i=0}^{n} Q_{n, i}^{2} R_{\ell+2^{i}-1}^{W} \\
& =\sum_{i=0}^{n} Q_{n, i}^{2} \sum_{x \in W} x^{\ell+2^{i}-1} \quad\left(\text { as } R_{\ell+2^{i}-1}^{W}=\sum_{x \in W} x^{\ell+2^{i}-1}\right) \\
& =\sum_{x \in W} x^{\ell-1} \sum_{i=0}^{n} Q_{n, i}^{2} x^{2^{i}} .
\end{aligned}
$$

It implies that

$$
V_{n+1} \sum_{i=0}^{n} Q_{n, i}^{2} \operatorname{tr}_{1, n+1}\left(x^{\ell+2^{i}-1}\right)=\sum_{x \in W} x^{\ell-1} V_{n+1} \sum_{i=0}^{n} Q_{n, i}^{2} x^{2^{i}} .
$$

On the other hand, $V_{n+1}\left(x_{1}, \ldots, x_{n}, x_{n+1}\right)=V_{n+1}\left(x_{1}, \ldots, x_{n}, x\right)$ for all $x \in W$; so, by [5, Proposition 2.6], we get

$$
V_{n+1}=\sum_{i=0}^{n} Q_{n, i}^{2} x^{2^{i}} \quad \text { for all } x \in W
$$

As a consequence

$$
\begin{aligned}
V_{n+1} \sum_{i=0}^{n} Q_{n, i}^{2} t r_{1, n+1}\left(x^{\ell+2^{i}-1}\right) & =\sum_{x \in W} x^{\ell-1}\left(\sum_{i=0}^{n} Q_{n, i}^{2} x^{2^{i}}\right)^{2} \\
& =\sum_{x \in W} x^{\ell-1} \sum_{i=0}^{n} Q_{n, i}^{2} x^{2^{i+1}} \\
& =\sum_{x \in W} \sum_{i=0}^{n} Q_{n, i}^{2} x^{\ell+2^{i+1}-1} \\
& =\sum_{i=0}^{n} Q_{n, i}^{2} \sum_{x \in W} x^{\ell+2^{i+1}-1} \\
& =\sum_{i=0}^{n} Q_{n, i}^{2} R_{\ell+2^{i+1}-1}^{W} \\
& =R_{\ell+2^{n+1}-1}^{W}+\sum_{i=0}^{n-1} Q_{n, i}^{2} R_{\ell+2^{i+1}-1}^{W} .
\end{aligned}
$$


Therefore, we get (b).

Proof of Theorem 3.1 for convenience, we denote

$$
\begin{aligned}
Q^{\left(s_{0}, s_{1}, \ldots, s_{n-1}\right)} & =Q_{n, 0}^{s_{0}} Q_{n, 1}^{s_{1}} \ldots Q_{n, n-1}^{s_{n}-1}, \\
C\left(s_{0}, s_{1}, \ldots, s_{n-1}\right) & =\frac{\left(\sum_{i=0}^{n-1} s_{i}-1\right) !}{\left(s_{0}-1\right) ! s_{1} ! \ldots s_{n-1} !} .
\end{aligned}
$$

Here $C\left(s_{0}, s_{1}, \ldots, s_{n-1}\right)=0$ if one of the numbers $\left(s_{0}-1\right), s_{1}, \ldots, s_{n-1}$ is negative. We see that

$$
\begin{aligned}
C\left(s_{0}, s_{1}, \ldots, s_{n-1}\right) & =\left(\sum_{i=0}^{n-1} s_{i}-1\right) \frac{\left(\sum_{i=0}^{n-1} s_{i}-2\right) !}{\left(s_{0}-1\right) ! s_{1} ! \ldots s_{n-1} !} \\
& =\frac{\left(\sum_{i=0}^{n-1} s_{i}-2\right) !}{\left(s_{0}-2\right) ! s_{1} ! \ldots s_{n-1} !}+\sum_{i=1}^{n-1} \frac{\left(\sum_{i=0}^{n-1} s_{i}-2\right) !}{\left(s_{0}-1\right) ! \ldots\left(s_{i}-1\right) \ldots s_{n-1} !} \\
& =\sum_{i=0}^{n-1} C\left(s_{0}, \ldots, s_{i}-1, \ldots, s_{n-1}\right) .
\end{aligned}
$$

For $0 \leq i \leq n-1$, setting $d_{i}=\operatorname{deg} Q_{n, i}$, we get $d_{i}=2^{n}-2^{i}$. So, the theorem is equivalent to

$$
\operatorname{tr}_{1, n}\left(x^{k}\right)=\sum C\left(s_{0}, s_{1}, \ldots, s_{n-1}\right) Q^{\left(s_{0}, s_{1}, \ldots, s_{n-1}\right)},
$$

where the sum is over all sequences $s_{0}, s_{1}, \ldots, s_{n-1}$ such that $\sum_{i=0}^{n-1} d_{i} s_{i}=k$.

The proposition is proved by induction on $k$.

Case $k<2^{n}-1$. We have

$$
\sum_{i=0}^{n-1} d_{i} s_{i}=k \Longleftrightarrow\left(2^{n}-1\right) s_{0}+\sum_{i=1}^{n-1} s_{i}=k \Longrightarrow s_{0}=0 .
$$

Then $C\left(s_{0}, s_{1}, \ldots, s_{n-1}\right)=0$ for all sequences $\left(s_{0}, \ldots, s_{n-1}\right)$ such that $\sum_{i=0}^{n-1} d_{i} s_{i}$ $=k$. As a consequence, the right hand side of (1) is equal to zero. On the other hand, by Lemma 3.2, $\operatorname{tr}_{1, n}\left(x^{k}\right)=0$ as $k<2^{n}-1$. Therefore, we get (1) for this case. 
Case $k \geq 2^{n}-1$. Suppose inductively that the theorem holds at degree less than or equal $k$. We have

$$
\begin{aligned}
& \sum_{d_{0} s_{0}+\cdots+d_{n-1} s_{n-1}=k+1} C\left(s_{0}, s_{1}, \ldots, s_{n-1}\right) Q^{\left(s_{0}, s_{1}, \ldots, s_{n-1}\right)} \\
= & \sum_{\sum d_{j} s_{j}=k+1} \sum_{i=0}^{n-1} C\left(s_{0}, \ldots, s_{i}-1, \ldots, s_{n-1}\right) Q^{\left(s_{0}, s_{1}, \ldots, s_{n-1}\right)} \\
= & \sum_{i=0}^{n-1} \sum_{d_{j} s_{j}=k+1} C\left(s_{0}, \ldots, s_{i}-1, \ldots, s_{n-1}\right) Q^{\left(s_{0}, s_{1}, \ldots, s_{n-1}\right)} \\
= & \left.\sum_{i=0}^{n-1} Q_{n, i} \sum_{\sum d_{j} s_{j}-d_{i}=k+1-\left(2^{n}-2^{i}\right)}, \ldots, s_{i}-1, \ldots, s_{n-1}\right) Q^{\left(s_{0}, \ldots, s_{i}-1, \ldots, s_{n-1}\right)} \\
= & \sum_{i=0}^{n-1} Q_{n, i} \operatorname{tr}_{1, n}\left(x^{k+1-\left(2^{n}-2^{i}\right)}\right)
\end{aligned}
$$

[by inductive hypothesis since $k+1-\left(2^{n}-2^{i}\right) \leq k$ ]

$=\operatorname{tr}_{1, n}\left(x^{k+1}\right) \quad($ by Lemma 3.3).

So, (1) holds at degree $k+1$. Therefore, the theorem is proved.

\section{Multilinear and alternating invariants}

Recall that $\mathbb{U}$ and $\mathbb{V}$ are vector spaces of dimensions $m$ and $n$ respectively with $m<n, \operatorname{tr}_{\mathbb{U}, \mathbb{V}}=\sum_{\mathbb{K}} \pi_{\mathbb{K}}^{*}: H^{*}(\mathbb{U})^{G L(\mathbb{U})} \rightarrow H^{*}(\mathbb{V})^{G L(\mathbb{V})}$, where the sum runs over all the subspaces $\mathbb{K}$ of dimension $(\operatorname{dim} \mathbb{V}-\operatorname{dim} \mathbb{U})$ in $\mathbb{V}$. Let $x_{1}, \ldots, x_{m}$ be a basis of $\mathbb{U}^{*}$. Then $H^{*}(\mathbb{U})=S\left(\mathbb{U}^{*}\right)=\mathbb{F}_{2}\left[x_{1}, \ldots, x_{m}\right]$. For each $f\left(x_{1}, \ldots, x_{m}\right) \in \mathbb{F}_{2}\left[x_{1}, \ldots, x_{m}\right]$, we define by $f$ the map

$$
\underbrace{\mathbb{V}^{*} \times \cdots \times \mathbb{V}^{*}}_{m \text { times }} \rightarrow S\left(\mathbb{V}^{*}\right), \quad\left(t_{1}, \ldots, t_{m}\right) \mapsto f\left(t_{1}, \ldots, t_{m}\right)
$$

Definition 4.1 For $m<n$, a polynomial $f\left(x_{1}, \ldots, x_{m}\right) \in \mathbb{F}_{2}\left[x_{1}, \ldots, x_{m}\right]$ is called multilinear (alternating) on $\mathbb{V}^{*}$ if the map $f$ is $m$ - multilinear (alternating).

Lemma 4.2 If $f\left(x_{1}, \ldots, x_{m}\right) \in \mathbb{F}_{2}\left[x_{1}, \ldots, x_{m}\right]$ is multilinear and alternating, then $f\left(x_{1}, \ldots, x_{m}\right)$ is a $G L\left(m, \mathbb{F}_{2}\right)$-invariant.

Proof For each $\omega \in G L\left(m, \mathbb{F}_{2}\right)$, we have

$$
\omega f\left(x_{1}, \ldots, x_{m}\right)=f\left(\omega x_{1}, \ldots, \omega x_{m}\right) .
$$


Since $f\left(x_{1}, \ldots, x_{m}\right)$ is is multilinear and alternating, we get

$$
f\left(\omega x_{1}, \ldots, \omega x_{m}\right)=(\operatorname{det} \omega) f\left(x_{1}, \ldots, x_{m}\right) .
$$

As $\operatorname{det} \omega=1$, we obtain $f\left(\omega x_{1}, \ldots, \omega x_{m}\right)=f\left(x_{1}, \ldots, x_{m}\right)$. Therefore $\omega f\left(x_{1}, \ldots, x_{m}\right)=f\left(x_{1}, \ldots, x_{m}\right)$. It follows that $f\left(x_{1}, \ldots, x_{m}\right)$ is a $G L\left(m, \mathbb{F}_{2}\right)$ invariant.

Definition 4.3 For each set of $m$ distinct non-negative numbers $\beta=\left\{\beta_{1}, \ldots, \beta_{m}\right\}$, let $\Sigma_{m}$ be the symmetric group on $\{1, \ldots, m\}$ and let

$$
\omega_{\beta}\left(x_{1}, \ldots, x_{m}\right)=\sum_{\sigma \in \Sigma_{m}} x_{1}^{2^{\beta_{\sigma(1)}}} \ldots x_{m}^{2^{\beta_{\sigma(m)}}} .
$$

This sum is invariant under $\Sigma_{m}$.

Lemma $4.4(i) \omega_{\beta}\left(x_{1}, \ldots, x_{m}\right)$ is multilinear and alternating.

(ii) $\omega_{\beta}\left(x_{1}, \ldots, x_{m}\right)$ is a $G L\left(m, \mathbb{F}_{2}\right)$-invariant.

Note that, for each $d>0$, in [4, Definition 2.1], Hưng and Peterson defined $\omega_{m}(d)=\sum x_{1}^{i_{1}} \ldots x_{m}^{i_{m}}$, where the sum is over all sequence $i_{1}, \ldots, i_{m}$ with $i_{j}$ either 0 or a power of 2 and $\sum i_{j}=d$. If $d$ is the degree of $\omega_{\beta}\left(x_{1}, \ldots, x_{m}\right)$, let $\alpha(d)$ be the number 1's occurring in the dyadic expansion of $d$, then $d=2^{\beta_{1}}+\cdots+2^{\beta_{m}}$ is the dyadic expansion of $d$ and $\alpha(d)=m$. Therefore, in this case, $\omega_{\beta}\left(x_{1}, \ldots, x_{m}\right)$ is actually the element $\omega_{m}(d)$ in [4, Definition 2.1]. However if $\alpha(d)<m$ then $\omega_{m}(d)$ maybe a $G L\left(m, \mathbb{F}_{2}\right)$-invariant but not multilinear and alternating. For example, let $m=2$ and $d=2$ we get $\omega_{2}(d)=Q_{2,1}\left(x_{1}, x_{2}\right)=x_{1}^{2}+x_{1} x_{2}+x_{2}^{2}$ is neither multilinear nor alternating, since $Q_{2,1}\left(x_{1}+x_{3}, x_{2}\right)=Q_{2,1}\left(x_{1}, x_{2}\right)+x_{2} x_{3}+x_{3}^{2} \neq$ $Q_{2,1}\left(x_{1}, x_{2}\right)+Q_{2,1}\left(x_{3}, x_{2}\right)$ and $Q\left(x_{1}, x_{1}\right)=x_{1}^{2} \neq 0$.

Proof of Lemma 4.4 The part (ii) is a corollary of [4, Theorem 2.2], where $\omega_{\beta}\left(x_{1}, \ldots, x_{m}\right)=\omega_{m}(d)$ with $\alpha(d)=k$. On the other hand, the part (ii) is also a corollary of the part (i) and Lemma 4.2. So, we only need to show the part (i).

We have $\omega_{\beta}\left(x_{1}, \ldots, x_{m}\right)$ is symmetric, so it is enough to show that the map $t_{1} \mapsto \omega_{\beta}\left(t_{1}, \ldots, t_{m}\right)$ is linear and $\omega_{\beta}\left(t_{1}, t_{2} \ldots, t_{m}\right)=0$ when $t_{1}=t_{2}$.

If $t_{1}=t_{11}+t_{12}$ then $t_{1}^{2^{a_{1}}}=t_{11}^{2^{a_{1}}}+t_{12}^{2^{a_{1}}}$. Thus

$$
\begin{aligned}
\omega_{\beta}\left(t_{11}+t_{12}, t_{2} \ldots, t_{m}\right) & =\sum\left(t_{11}+t_{12}\right)^{2^{a_{1}}} t_{2}^{2^{a_{2}}} \ldots t_{m}^{2^{a_{m}}} \\
& =\sum t_{11}^{2^{a_{1}}} t_{2}^{2^{a_{2}}} \ldots t_{m}^{2^{a_{m}}}+\sum t_{12}^{2^{a_{1}}} t_{2}^{2^{a_{2}}} \ldots t_{m}^{2^{a_{m}}} \\
& =\omega_{\beta}\left(t_{11}, t_{2} \ldots, t_{m}\right)+\omega_{\beta}\left(t_{12}, t_{2} \ldots, t_{m}\right) .
\end{aligned}
$$

Being lax on notation to avoid cumbersome formulas, we rewrite $\omega_{\beta}\left(t_{1}, t_{2}, \ldots, t_{m}\right)$ as

$$
\omega_{\beta}\left(t_{1}, t_{2}, \ldots, t_{m}\right)=\sum_{a_{1}<a_{2}}\left(t_{1}^{2^{a_{1}}} t_{2}^{2^{a_{2}}}+t_{1}^{2^{a_{2}}} t_{2}^{2^{a_{1}}}\right) t_{3}^{2^{a_{3}}} \ldots t_{m}^{2^{a_{m}}} .
$$


Thus, if $t_{1}=t_{2}$ then $\left(t_{1}^{2^{a_{1}}} t_{2}^{2^{a_{2}}}+t_{1}^{2^{a_{2}}} t_{2}^{2^{a_{1}}}\right)=2 t_{1}^{2^{a_{1}}} t_{2}^{2^{a_{2}}}=0$. So $\omega_{\beta}\left(t_{1}, t_{1} \ldots, t_{m}\right)=0$. The lemma is proved.

The following proposition is also numbered as Proposition 1.3 in the introduction.

\section{Proposition 4.5 The set}

$$
\left\{\omega_{\beta}\left(x_{1}, \ldots, x_{m}\right) \mid \beta=\left(\beta_{1}, \ldots, \beta_{m}\right) \in \mathbb{N}^{m}, \beta_{i} \neq \beta_{j} \quad \text { for all } i \neq j\right\}
$$

forms a basis for the subspace of $\mathbb{F}_{2}\left[x_{1}, \ldots, x_{m}\right]$ generated by multilinear and alternating invariants.

Proof For each $\beta=\left(\beta_{1}, \ldots, \beta_{m}\right) \in \mathbb{N}^{m}$ with $\beta_{i} \neq \beta_{j}$ for all $i \neq j$, the sum $2^{\beta_{1}}+\cdots+2^{\beta_{m}}$ is the dyadic expansion of $\operatorname{deg} \omega_{\beta}\left(x_{1}, \ldots, x_{m}\right)$. So, in each degree $d$, there exists at most an element of the form $\omega_{\beta}\left(x_{1}, \ldots, x_{m}\right)$ with $d=\sum_{j=1}^{m} 2^{s_{j}}$. Therefore the set of elements $\omega_{\beta}\left(x_{1}, \ldots, x_{m}\right)$ is linearly independent.

Suppose that $Q\left(x_{1}, \ldots, x_{m}\right)$ is a multilinear and alternating invariant of degree $d$ in $D_{m}$. We prove that $Q\left(x_{1}, \ldots, x_{m}\right)=\omega_{\beta}\left(x_{1}, \ldots, x_{m}\right)$ for some $\beta=\left(\beta_{1}, \ldots, \beta_{m}\right)$ such that $\beta_{i} \neq \beta_{j}$ for all $i \neq j$ and $d=2^{\beta_{1}}+\cdots+2^{\beta_{m}}$. Let $x_{1}^{i_{1}} x_{2}^{i_{2}} \ldots x_{m}^{i_{m}}$ be an arbitrary monomial of $Q\left(x_{1}, \ldots, x_{m}\right)$. We write $Q\left(x_{1}, \ldots, x_{m}\right)$ as

$$
Q\left(x_{1}, \ldots, x_{m}\right)=x_{1}^{i_{1}} x_{2}^{i_{2}} \ldots x_{m}^{i_{m}}+\sum_{\left(j_{1}, j_{2}, \ldots, j_{m}\right) \neq\left(i_{1}, i_{2}, \ldots, i_{m}\right)} x_{1}^{j_{1}} x_{2}^{j_{2}} \ldots x_{m}^{j_{m}} .
$$

Suppose that $i_{1}=0$. Since $Q\left(x_{1}, \ldots, x_{m}\right)$ is multilinear, by Definition 4.1, we get $Q\left(x_{1}+x_{n}, \ldots, x_{m}\right)=Q\left(x_{1}, \ldots, x_{m}\right)+Q\left(x_{n}, \ldots, x_{m}\right)$. So

$$
\begin{aligned}
& x_{2}^{i_{2}} \ldots x_{m}^{i_{m}} \\
& =\sum_{\left(j_{1}, \ldots, j_{m}\right) \neq\left(i_{1}, \ldots, i_{m}\right)}\left[\left(x_{1}+x_{n}\right)^{j_{1}} x_{2}^{j_{2}} \ldots x_{m}^{j_{m}}+x_{1}^{j_{1}} x_{2}^{j_{2}} \ldots x_{m}^{j_{m}}+x_{n}^{j_{1}} x_{2}^{j_{2}} \ldots x_{m}^{j_{m}}\right] \\
& \quad=\sum_{j_{1}=0} x_{2}^{j_{2}} \ldots x_{m}^{j_{m}}+\sum_{j_{1}>0} \sum_{j=1}^{j_{1}-1}\left(\begin{array}{c}
j_{1} \\
j
\end{array}\right) x_{1}^{i} x_{2}^{j_{2}} \ldots x_{m}^{j_{m}} x_{n}^{j_{1}-j} .
\end{aligned}
$$

This is a contradiction as $\left(j_{2}, \ldots, j_{m}\right) \neq\left(i_{2}, \ldots, i_{m}\right)$. Thus $i_{1} \neq 0$. Since $Q\left(x_{1}+\right.$ $\left.x_{n}, \ldots, x_{m}\right)=Q\left(x_{1}, \ldots, x_{m}\right)+Q\left(x_{n}, \ldots, x_{m}\right)$ again, we get

$$
\sum_{i=1}^{i_{1}-i}\left(\begin{array}{c}
i_{1} \\
i
\end{array}\right) x_{1}^{i} x_{2}^{i_{2}} \ldots x_{m}^{i_{m}} x_{n}^{i_{1}-i}=\sum_{j_{1}=0} x_{2}^{j_{2}} \ldots x_{m}^{j_{m}}+\sum_{j_{1}>0} \sum_{j=1}^{j_{1}-1}\left(\begin{array}{c}
j_{1} \\
j
\end{array}\right) x_{1}^{j} x_{2}^{j_{2}} \ldots x_{m}^{j_{m}} x_{n}^{j_{1}-j}
$$

If $i_{1}$ is not a power of 2 , then $\left(\begin{array}{c}i_{1} \\ i\end{array}\right) \neq 0$ for some $1 \leq i \leq i_{1}$. Therefore, there exists a $(m+1)$-tuple $\left(j, j_{1}, j_{2}, \ldots, j_{m}\right)$ with $j_{1}>0,1 \leq j \leq j_{1}-1$ and $\left(\begin{array}{c}j_{1} \\ j\end{array}\right) \neq 0$ such that $\left(i, i_{2}, \ldots, i_{m}, i_{1}-i\right)=\left(j, j_{2}, \ldots, j_{m}, j_{1}-j\right)$. It follows that $\left(i_{1}, \ldots, i_{m}\right)=$ $\left(j_{1}, \ldots, j_{m}\right)$. This is a contradiction. Hence $i_{1}$ is a power of 2 . Similarly, we also get 
$i_{2}, \ldots, i_{m}$ are powers of 2 . We write $i_{1}=2^{\beta_{1}}, i_{2}=2^{\beta_{2}}, \ldots, i_{m}=2^{\beta_{m}}$ for some nonnegative integers $\beta_{1}, \beta_{2}, \ldots, \beta_{m}$. Now, we show that $i_{s} \neq i_{t}$ for all $s \neq t$. Without lost of generality, suppose to the contrary that $i_{1}=i_{2}$. As $Q\left(x_{1}, x_{2}, \ldots, x_{m}\right) \in D_{m}$, it is invariant under the transposition of $x_{1}$ and $x_{2}$ that keeps the other variables fixed. It follows that $Q\left(x_{1}, x_{2}, \ldots, x_{m}\right)$ has the form

$$
x_{1}^{i_{1}} x_{2}^{i_{1}} x_{3}^{i_{3}} \ldots x_{m}^{i_{m}}+\sum_{j_{1}=j_{2} \neq i_{1}} x_{1}^{j_{1}} x_{2}^{j_{2}} x_{3}^{j_{3}} \ldots x_{m}^{j_{m}}+\sum_{j_{1} \neq j_{2}}\left(x_{1}^{j_{1}} x_{2}^{j_{2}}+x_{1}^{j_{2}} x_{2}^{j_{1}}\right) x_{3}^{j_{3}} \ldots x_{m}^{j_{m}} .
$$

On the other hand $Q\left(x_{1}, x_{2}, \ldots, x_{m}\right)$ is alternating, by Definition 4.1 , we get $Q\left(x_{1}, x_{1}, \ldots, x_{m}\right)=0$. So

$$
x_{1}^{2 i_{1}} x_{3}^{i_{3}} \ldots x_{m}^{i_{m}}+\sum_{j_{1}=j_{2}} x_{1}^{j_{1}+j_{2}} x_{3}^{j_{3}} \ldots x_{m}^{j_{m}}=0
$$

Hence, there is a tuple $\left(j_{1}, j_{2}, \ldots, j_{m}\right)$ such that $j_{1}=j_{2}, j_{1}+j_{2}=2 i_{1}, j_{k}=$ $i_{k}$ for $k \geq 3$. It implies that $\left(i_{1}, i_{2}, i_{3}, \ldots, i_{m}\right)=\left(j_{1}, j_{2}, j_{3}, \ldots, j_{m}\right)$. This is a contradiction. Therefore $i_{1} \neq i_{2}$. Similarly, we get $i_{s} \neq i_{t}$ for all $s \neq t$.

It has shown that if $x_{1}^{i_{1}} x_{2}^{i_{2}} \ldots x_{m}^{i_{m}}$ is a monomial of $Q\left(x_{1}, x_{2}, \ldots, x_{m}\right)$ then $i_{j}=2^{\beta_{j}}$ for $1 \leq j \leq m$ and $\beta_{i} \neq \beta_{j}$ for $i \neq j$. As $Q\left(x_{1}, x_{2}, \ldots, x_{m}\right)$ is invariant under the symmetric group $\Sigma_{m}$, it contains all permutations of $x_{1}^{i_{1}} x_{2}^{i_{2}} \ldots x_{m}^{i_{m}}$ as terms. Since $\beta_{1}, \ldots, \beta_{m}$ are distinct, the sum of powers of $x_{1}, \ldots, x_{m}$ in the monomial $x_{1}^{i_{1}} x_{2}^{i_{2}} \ldots x_{m}^{i_{m}}, 2^{\beta_{1}}+2^{\beta_{2}}+\cdots+2^{\beta_{m}}$, is the the dyadic expansion of $\operatorname{deg} Q\left(x_{1}, x_{2}, \ldots, x_{m}\right)$. Therefore $Q\left(x_{1}, x_{2}, \ldots, x_{m}\right)$ only contains $x_{1}^{i_{1}} x_{2}^{i_{2}} \ldots x_{m}^{i_{m}}$ and its permutations as terms. Let $\beta=\left(\beta_{1}, \beta_{2}, \ldots, \beta_{m}\right)$, we get

$$
Q\left(x_{1}, x_{2}, \ldots, x_{m}\right)=\omega_{\beta}\left(x_{1}, x_{2}, \ldots, x_{m}\right)
$$

So, the proposition is proved.

As we had known that $\omega_{\beta}\left(x_{1}, \ldots, x_{m}\right)$ is $\omega_{m}(d)$ with $\alpha(d)=m$ in $[4$, Definition 2.1], where $d$ is the degree of $\omega_{\beta}\left(x_{1}, \ldots, x_{m}\right)$, the following is a corollary of [4, Corollary 2.7].

Corollary 4.6 Suppose that $\beta=\left\{\beta_{1}, \ldots, \beta_{m}\right\}$ with $\beta_{1}<\beta_{2}<\cdots<\beta_{m}$. Then $\left(Q_{m, 0}\right)^{2^{\beta_{1}}} \mid \omega_{\beta}\left(x_{1}, \ldots, x_{m}\right)$.

From Proposition 4.5 and Corollary 4.6, we get the following result.

Corollary 4.7 If $Q \in D_{m}$ is multilinear and alternating, then $Q_{m, 0}$ divides $Q$.

Example 4.8 (i) $Q_{m, 0}=\omega_{\beta}\left(x_{1}, \ldots, x_{m}\right)$ with $\beta=(0,1, \ldots, m-1)$.

(ii) For each $0 \leq i \leq m-1, Q_{m, 0} Q_{m, i}=\omega_{\beta}\left(x_{1}, \ldots, x_{m}\right)$ with $\beta=(0,2, \ldots$ $i-1, i+1, \ldots, m)$.

Proof From [4, Theorem 2.2], we have $Q_{m, 0}=x_{1}^{2^{m-1}} x_{2}^{2^{m-2}} \ldots x_{m-1}^{2} x_{m}+$ (symmetried). Then, we obtain (i). 
We have

$$
\begin{aligned}
Q_{m+1,0} & =Q_{m, 0} V_{m+1} \\
& =Q_{m, 0} \sum_{i=0}^{m-1} Q_{m, i} x_{m+1}^{2} \quad(\text { by [5, Proposition 2.6] ). }
\end{aligned}
$$

So, $Q_{m, 0} Q_{m, i}$ is the coefficient of $x_{m+1}^{2^{i}}$ in $Q_{m+1,0}$. On the other hand, from [4, Theorem 2.2] again, we get

$$
Q_{m+1,0}=x_{1}^{2^{m}} x_{2}^{2^{m-1}} \ldots x_{m}^{2} x_{m+1}+(\text { symmetried })
$$

So the coefficient of $x_{m+1}^{2^{i}}$ in $Q_{m+1,0}$ is $\sum_{\left(i_{1}, \ldots, i_{m}\right)} x_{1}^{i_{1}} x_{2}^{i_{2}} \ldots x_{m}^{i_{m}}$, where the sum is over all permutations of $\left(1, \ldots, 2^{i-1}, 2^{i+1}, \ldots, 2^{m}\right)$. As a consequence, we get

$$
Q_{m, 0} Q_{m, i}=\sum_{\left(i_{1}, \ldots, i_{m}\right)} x_{1}^{i_{1}} x_{2}^{i_{2}} \ldots x_{m}^{i_{m}}=\omega_{\beta}\left(x_{1}, \ldots, x_{m}\right)
$$

with $\beta=\left(0, \ldots, 2^{i-1}, 2^{i+1}, \ldots, 2^{m}\right)$. Thus, (ii) is proved.

\section{Transfer on powers of multilinear and alternating invariants}

In this section, we first study the transfer $t r_{m, n}$ in which $n=m+1$. Recall that $t r_{\mathbb{U}, \mathbb{V}}: H^{*}(\mathbb{U})^{G L(\mathbb{U})} \rightarrow H^{*}(\mathbb{V})^{G L(\mathbb{V})}$, or $t r_{m, m+1}: D_{m} \rightarrow D_{m+1}$, is given by

$$
\operatorname{tr}_{m, m+1}(Q)=\sum_{W \leq \mathbb{V}^{*}, \operatorname{dim} W=m} Q(W) .
$$

Let $x_{1}, \ldots, x_{m+1}$ be a basis of $\mathbb{V}^{*}$. For each $1 \leq j \leq m+1$, we denote by $X_{j}$ the subspace of $\mathbb{V}^{*}$ generated by $x_{1}, \ldots, x_{j-1}, x_{j+1}, \ldots, x_{m+1}$. Then, we have $\operatorname{dim} X_{j}=m$.

Lemma 5.1 There is an 1-1 correspondence between the set of all m-dimensional vector spaces $W$ of $\mathbb{V}^{*}$ and the set of $(m+1)$-tuple of scalars $\left(c_{1}, \ldots, c_{m+1}\right)$ in which not all $c_{i}$ are zero such that $Q(W)=c_{1} Q\left(X_{1}\right)+\cdots+c_{m+1}\left(X_{m+1}\right)$ for all multilinear and alternating invariants $Q$ of $D_{m}$.

Proof Suppose that $W$ is an $m$-dimensional vector space of $\mathbb{V}^{*}$ and $y_{1}, \ldots, y_{m}$ is a basis of $W$. We have $\left(y_{1} \ldots y_{m}\right)=\left(x_{1} \ldots x_{m+1}\right) C$ where $C$ is a $(m+1) \times m$-matrix and $\operatorname{rank} C=m$. So $y_{j}=\sum_{i=1}^{m+1} c_{i j} x_{i}$. Setting $c_{j}=\sum_{i_{\ell} \neq j \forall \ell, i_{k} \neq i_{\ell}} c_{i_{1}, 1} \ldots c_{i_{m+1}, m}$ for $1 \leq j \leq m+1$, for arbitrary multilinear and alternating $Q$ of $D_{m}$, we get

$$
Q(W)=Q\left(y_{1}, \ldots, y_{m}\right)=c_{1} Q\left(X_{1}\right)+\cdots+c_{m+1} Q\left(X_{m+1}\right) .
$$

Conversely, we show by induction on $m$ that for each $(m+1)$-tuple of scalars $\left(c_{1}, \ldots, c_{m+1}\right)$ in which not all $c_{i}$ are zero, there exists exactly one $m$-dimensional 
subspace $W$ of $\mathbb{V}^{*}$ satisfying $Q(W)=c_{1} Q\left(X_{1}\right)+\cdots+c_{m+1} Q\left(X_{m+1}\right)$ for all multilinear alternating invariants $Q$ of $D_{m}$.

For $m=1$, let $W=\mathbb{F}_{2}<c_{1} x_{2}+c_{2} x_{1}>$ we have $\operatorname{dim} W=1$ as $\left(c_{1}, c_{2}\right) \neq(0,0)$. For a multilinear alternating invariants $Q$ of $D_{1}$, we get $Q(W)=Q\left(c_{1} x_{2}+c_{2} x_{1}\right)=$ $c_{1} Q\left(x_{2}\right)+c_{2} Q\left(x_{1}\right)=c_{1} Q\left(X_{1}\right)+c_{2} Q\left(X_{2}\right)$ as $X_{1}=\mathbb{F}_{2}<x_{2}>, X_{2}=\mathbb{F}_{2}<x_{1}>$.

Let us consider the following two cases.

Case 1. There is index $i$ such that $c_{i}=0$. Setting $S=\left\{x_{1}, \ldots, x_{m+1}\right\}$ and $\mathbb{V}^{(i)}=$ $\mathbb{F}_{2}<S \backslash\left\{x_{i}\right\}>$. Then $\mathbb{V}^{(m+1)}=\mathbb{V}^{*}$ and $\operatorname{dim} \mathbb{V}^{(i)}=m$. For each $j \neq i$, we denote by $X_{j}^{(i)}$ the subspace of $\mathbb{V}^{(i)}$ generated by $S \backslash\left\{x_{i}, x_{j}\right\}$. We have $\operatorname{dim} X_{j}^{(i)}=m-1$. Applying the inductive hypothesis to $\mathbb{V}^{(i)}$, since not all $c_{j}$ are zero for $1 \leq j \leq$ $m+1, j \neq i$, there is one $(m-1)$-dimensional subspace $W^{(i)}$ of $\mathbb{V}^{(i)}$ such that

$$
Q_{m-1}\left(W^{(i)}\right)=\sum_{1 \leq j \leq m+1, j \neq i} c_{j} Q_{m-1}\left(X_{j}^{(i)}\right)
$$

for all multilinear alternating invariants $Q_{m-1}$ of $\mathbb{F}_{2}\left[x_{1}, \ldots\right.$, $\left.x_{i-1}, x_{i+1}, \ldots, x_{m+1}\right]^{G L\left(m, \mathbb{F}_{2}\right)}$. Let $W$ be the subspace generated by $W^{(i)} \cup\left\{x_{i}\right\}$. Since $W^{(i)} \leq \mathbb{F}_{2}\left[S \backslash\left\{x_{i}\right\}\right]$, we get $W=W^{(i)} \oplus \mathbb{F}_{2}<x_{i}>$ and $W$ is an $m$ dimensional subspace of $\mathbb{V}^{*}$. Suppose that $\left(\alpha_{1}, \ldots, \alpha_{m-1}\right)$ is a basis for $W^{(i)}$. As $W^{(i)} \leq \mathbb{F}_{2}\left\langle x_{1}, \ldots, x_{i-1}, x_{i+1}, \ldots, x_{m+1}\right\rangle$, each $\alpha_{j}$ can be written as

$$
\alpha_{j}=\sum_{1 \leq k \leq m+1, k \neq i} c_{k j} x_{k}
$$

Then

$$
\begin{aligned}
Q_{m-1}\left(W^{(i)}\right) & =Q_{m-1}\left(\alpha_{1}, \ldots \alpha_{m-1}\right) \\
& =\sum_{j=1}^{m+1}\left(\sum_{\sigma \in \Sigma_{\{1, \ldots, m+1\} \backslash\{i, j\}}} c_{\sigma(1), 1} \ldots c_{\sigma(m+1), m-1}\right) Q_{m-1}\left(X_{j}^{(i)}\right) .
\end{aligned}
$$

From the uniqueness of the subspace $W^{(i)}$ by the inductive hypothesis, we get

$$
c_{j}=\sum_{\sigma \in \Sigma_{\{1, \ldots, m+1\} \backslash\{i, j\}}} c_{\sigma(1), 1} \ldots c_{\sigma(m+1), m-1} \quad \text { for } 1 \leq j \leq m+1 .
$$

Now, for an arbitrary multilinear and alternating invariant $Q_{m}$ of $D_{m}$ we have

$$
\begin{aligned}
Q_{m}(W) & =Q_{m}\left(\alpha_{1}, \ldots, \alpha_{m-1}, x_{i}\right) \\
& =Q_{m}\left(\sum_{k \neq i} c_{k 1} x_{k}, \ldots, \sum_{k \neq i} c_{k(m-1)} x_{k}, x_{i}\right)
\end{aligned}
$$




$$
\begin{aligned}
& =\sum_{j=1}^{m+1}\left(\sum_{\sigma \in \Sigma_{\{1, \ldots, m+1\} \backslash\{i, j\}}} c_{\sigma(1), 1} \ldots c_{\sigma(m+1), m-1}\right) Q_{m}\left(X_{j}^{(i)}, x_{i}\right) \\
& =\sum_{j=1}^{m+1} c_{j} Q_{m}\left(X_{j}^{(i)}, x_{i}\right)=\sum_{j=1}^{m+1} c_{j} Q_{m}\left(X_{j}\right)
\end{aligned}
$$

as $Q_{m}\left(X_{j}^{(i)}, x_{i}\right)=Q_{m}\left(X_{j}\right)$. So we get the conclusion.

Case 2. $c_{i}=1$ for all $1 \leq i \leq m+1$. By the similar argumentation as in Case 1 for $\left(c_{1}, \ldots, c_{m}\right)$, there is one $(m-1)$-dimensional subspace $W^{(m+1)}$ of $\mathbb{F}_{2}\left\langle x_{1}, \ldots, x_{m}\right\rangle$ such that

$$
Q_{m-1}\left(W^{(m+1)}\right)=\sum_{1 \leq j \leq m} Q_{m-1}\left(X_{j}^{(m+1)}\right)
$$

for all multilinear alternating invariants $Q_{m-1}$ of $D_{m-1}$. We see that $x_{1} \notin W^{(m+1)}$. Indeed, if $x_{1} \in W^{(m+1)}$, let $\left(\alpha_{1}, \alpha_{2}, \ldots, \alpha_{m-1}\right)$ be a basis for $W^{(m+1)}$ such that $\alpha_{1}=x_{1}$ and $\alpha_{2}, \ldots, \alpha_{m-1} \in \mathbb{F}_{2}<x_{2}, \ldots, x_{m}>$. Then, the coefficient of $Q_{m-1}\left(X_{1}^{(m+1)}\right)$ in the expansion of $Q_{m-1}\left(W^{(m+1)}\right)=Q\left(x_{1}, \alpha_{2}, \ldots, \alpha_{m-1}\right)$ is equal to 0 . This contradicts to $c_{1}=1$. So $x_{1} \notin W^{(m+1)}$. Similarly, we can prove that $x_{i} \notin W^{(m+1)}$ for all $1 \leq i \leq m$.

Now let $W$ be the subspace of $\mathbb{V}^{*}$ generated by $W^{(m+1)}$ and $x_{1}+x_{m+1}$. Since $x_{m+1} \notin W^{(m+1)}$ it follows that $\operatorname{dim} W=m$. Suppose that $\left(\alpha_{1}, \ldots, \alpha_{m-1}\right)$ is a basis of $W^{(m+1)}$ and $\alpha_{j}=\sum_{k=1}^{m} c_{k j} x_{k}$. By the similar argumentation as in Case 1, we get

$$
c_{j}=\sum_{\sigma \in \Sigma_{\{1, \ldots, m\} \backslash\{j\}}} c_{\sigma(1), 1} \ldots c_{\sigma(m), m-1} \quad \text { for } 1 \leq j \leq m .
$$

Hence, for any multilinear and alternating invariant $Q_{m}$ of $D_{m}$, we obtain

$$
\begin{aligned}
Q(W)= & Q\left(\alpha_{1}, \ldots, \alpha_{m-1}, x_{1}+x_{m+1}\right) \\
= & Q\left(\alpha_{1}, \ldots, \alpha_{m-1}, x_{1}\right)+Q\left(\alpha_{1}, \ldots, \alpha_{m-1}, x_{m+1}\right) \\
= & \sum_{j=1}^{m} c_{j} Q\left(X_{j}^{(m+1)}, x_{1}\right)+\sum_{j=1}^{m} c_{j} Q\left(X_{j}^{(m+1)}, x_{m+1}\right) \\
= & c_{1} Q\left(X_{1}^{(m+1)}, x_{1}\right)+\sum_{j=1}^{m} c_{j} Q\left(X_{j}^{(m+1)}, x_{m+1}\right) \\
& {\left[\text { as } Q\left(X_{j}^{(m+1)}, x_{1}\right)=0 \text { for all } j \neq 1\right] }
\end{aligned}
$$




$$
\begin{aligned}
& =c_{1} Q\left(X_{m+1}\right)+\sum_{j=1}^{m} c_{j} Q\left(X_{j}\right) \quad\left[\text { as } Q\left(X_{1}^{(m+1)}, x_{1}\right)=Q\left(X_{m+1}\right)\right] \\
& =Q\left(X_{1}\right)+\cdots+Q\left(X_{m+1}\right) \quad\left(\text { as } c_{j}=1 \quad \text { for all } j\right) .
\end{aligned}
$$

The lemma follows.

The following theorem is also numbered as Theorem 1.4 in the introduction.

Theorem 5.2 Suppose that $Q$ is a multilinear and alternating invariant in $D_{m}$. Let $y_{j}=Q\left(X_{j}\right)$ for $j=1, \ldots, m+1$. Then, for $r>0$ we have

$$
t r_{m, m+1}\left(Q^{r}\right)=\sum \frac{\left(s_{0}+\cdots+s_{m}-1\right) !}{\left(s_{0}-1\right) ! s_{1} ! \ldots s_{m} !} Q_{m+1,0}^{s_{0}} \ldots Q_{m+1, m}^{s_{m}}\left(y_{1}, \ldots, y_{m+1}\right)
$$

summed over all sequences of non-negative intergers $\left(s_{0}, \ldots, s_{m}\right)$ such that

$$
\sum_{i=0}^{m}\left(2^{m+1}-2^{i}\right) s_{i}=r
$$

Proof By the definition of $t r_{m, m+1}$, we have

$$
t r_{m, m+1}\left(Q^{r}\right)=\sum_{W \leq \mathbb{V}^{*}, \operatorname{dim} W=m} Q^{r}(W) .
$$

Following Lemma 5.1 and the definition of $t r_{1, m+1}$, it follows that

$$
\begin{aligned}
t r_{m, m+1}\left(Q^{r}\right) & =\sum_{c_{1}, \ldots, c_{m+1} \in \mathbb{F}_{2}}\left(c_{1} Q\left(X_{1}\right)+\cdots+c_{m+1} Q\left(X_{m+1}\right)\right)^{r} \\
& =\sum_{c_{1}, \ldots, c_{m+1} \in \mathbb{F}_{2}}\left(c_{1} y_{1}+\cdots+c_{m+1} y_{m+1}\right)^{r} \\
& =t r_{1, m+1} y_{1}^{r} .
\end{aligned}
$$

Now, applying Theorem 3.1, we get the theorem.

The following is numbered as Corollary 1.5 in the introduction.

Corollary 5.3 (i) $t r_{m, m+1}\left(Q_{m, 0}^{j}\right)=0$ for $0<j<2^{m+1}-1$.

(ii) $t r_{m, m+1}\left(Q_{m, 0}^{2^{m+1}-1}\right)=Q_{m+1,0}^{2^{m}-1}$.

Proof By Example 4.8(i), $Q_{m, 0}$ is multilinear and alternating. So, we apply Theorem 5.2 to compute $t r_{m, m+1}\left(Q_{m, 0}^{r}\right)$.

(i) We consider the equation $\sum_{i=0}^{m}\left(2^{m+1}-2^{i}\right) s_{i}=r$.

If $r$ is odd then $s_{0} \geq 1$. We have $\sum_{i=0}^{m}\left(2^{m+1}-2^{i}\right) s_{i} \geq 2^{m+1}-1$. But $0<r<2^{m+1}-1$. It follows that the equation has no solution. Therefore 
$t r_{m, m+1}\left(Q_{m, 0}^{r}\right)=0$. If $r$ is even, we write $r=2^{k}$. $\ell$ where $k, \ell \in \mathbb{N}$ and $\ell$ odd. We have $t r_{m, m+1}\left(Q_{m, 0}^{r}\right)=\left(t r_{m, m+1}\left(Q_{m, 0}^{\ell}\right)\right)^{2^{k}}$. Since $0<r<2^{m+1}-1$ we get $0<\ell<2^{m+1}-1$. So, applying the above case, we obtain $t r_{m, m+1}\left(Q_{m, 0}^{\ell}\right)=0$. Hence $t r_{m, m+1}\left(Q_{m, 0}^{r}\right)=0$. Part (i) of the corollary is proved.

(ii) We have the equation $\sum_{i=0}^{m}\left(2^{m+1}-2^{i}\right) s_{i}=2^{m+1}-1$ has exactly one solution, namely $s_{0}=1, s_{j}=0$ for $1 \leq j \leq m$. By Theorem 5.2, we get

$$
t r_{m, m+1}\left(Q_{m, 0}^{2^{m+1}-1}\right)=Q_{m+1,0}\left(y_{1}, \ldots, y_{m+1}\right)
$$

where $y_{j}=Q\left(x_{1}, \ldots, x_{j-1}, x_{j+1}, \ldots, x_{m+1}\right)$ for $1 \leq j \leq m+1$. Furthermore, from [4, Theorem 2.2] we have

$$
Q_{m+1,0}\left(y_{1}, \ldots, y_{m+1}\right)=y_{1}^{2^{m}} y_{2}^{2^{m-1}} \ldots y_{m}^{2} y_{m+1}+(\text { symmetried }) .
$$

We will use Lemma 6.2 to find invariant monomials of $Q_{m+1,0}\left(y_{1}, \ldots, y_{m+1}\right)$. First, we show by induction on $m$ that the maximal monomial in variables $x_{1}, \ldots, x_{m+1}$ of $Q_{m+1,0}\left(y_{1}, \ldots, y_{m+1}\right)$, by Definition 6.1 , is

$$
x_{1}^{2^{m}-1} x_{2}^{2\left(2^{m}-1\right)} \ldots x_{m}^{2^{m-1}\left(2^{m}-1\right)} x_{m+1}^{2^{m}\left(2^{m}-1\right)}
$$

For $m=1$, we have $y_{1}=Q_{1,0}\left(x_{2}\right)=x_{2}, y_{2}=Q_{1,0}\left(x_{1}\right)=x_{2}$. So $Q_{2,0}\left(y_{1}, y_{2}\right)=y_{1}^{2} y_{2}+y_{1} y_{2}^{2}=x_{1} x_{2}^{2}+x_{1}^{2} x_{2}$. Hence, the maximal monomial of $Q_{2,0}\left(y_{1}, y_{2}\right)$ is $x_{1} x_{2}^{2}$.

Now we consider $Q_{m+1,0}\left(y_{1}, \ldots, y_{m+1}\right)$. From the inductive definitions of the Dickson invariants and the Mùi invariants in $[2,5]$, for $i=1, \ldots, m$, we have

$$
\begin{aligned}
y_{i} & =Q_{m, 0}\left(x_{1}, \ldots, x_{i-1}, x_{i+1}, \ldots, x_{m+1}\right) \\
& =Q_{m-1,0}\left(x_{1}, \ldots, x_{i-1}, x_{i+1}, \ldots, x_{m}\right) V_{m}\left(x_{1}, \ldots, x_{i-1}, x_{i+1}, \ldots, x_{m+1}\right) \\
& =\sum_{j=1}^{m-1}\left(Q_{m-1,0} Q_{m-1, j}\right)\left(x_{1}, \ldots, x_{i-1}, x_{i+1}, \ldots, x_{m}\right) x_{m+1}^{2^{j}}
\end{aligned}
$$

(applying [5, Proposition 2.6] ).

Thus, the maximal monomial of each $y_{i}$ is a term of the polynomial

$$
Q_{m-1,0}\left(x_{1}, \ldots, x_{i-1}, x_{i+1}, \ldots, x_{m}\right) x_{m+1}^{2^{m-1}}
$$

It should be noted that $y_{m+1}=Q_{m, 0}\left(x_{1}, \ldots, x_{m}\right)$; so, the power of $x_{m+1}$ in $y_{m+1}$ equals to zero. For $1 \leq i \leq m$, denoting

$$
y_{i}^{\prime}=Q_{m-1,0}\left(x_{1}, \ldots, x_{i-1}, x_{i+1}, \ldots, x_{m}\right) .
$$


Then, we get

$$
\begin{aligned}
& Q_{m+1,0}\left(y_{1}, \ldots, y_{m+1}\right) \\
& \quad=Q_{m, 0}^{2}\left(y_{1}^{\prime}, \ldots, y_{m}^{\prime}\right) y_{m+1} x_{m+1}^{2^{m-1}\left(2^{m}+2^{m-1}+\cdots+2\right)}+(\text { smaller terms }) \\
& =Q_{m, 0}^{2}\left(y_{1}^{\prime}, \ldots, y_{m}^{\prime}\right) x_{1} x_{2}^{2} \ldots x_{m}^{2^{m-1}} x_{m+1}^{2^{m}\left(2^{m}-1\right)}+(\text { smaller terms }),
\end{aligned}
$$

as $y_{m+1}=x_{1} x_{2}^{2} \ldots x_{m}^{2^{m-1}}+($ smaller terms). On the other hand, by the inductive hypothesis, we have

$Q_{m, 0}\left(y_{1}^{\prime}, \ldots, y_{m}^{\prime}\right)=x_{1}^{2^{m-1}-1} x_{2}^{2\left(2^{m-1}-1\right)} \ldots x_{m}^{2^{m-1}\left(2^{m-1}-1\right)}+($ smaller terms $)$.

Therefore

$$
\begin{aligned}
Q_{m+1,0}\left(y_{1}, \ldots, y_{m+1}\right)= & x_{1}^{2^{m}-1} x_{2}^{2\left(2^{m}-1\right)} \ldots x_{m}^{2^{m-1}\left(2^{m}-1\right)} x_{m+1}^{2^{m}\left(2^{m}-1\right)} \\
& +(\text { smaller terms }) .
\end{aligned}
$$

So, the maximal monomial of $Q_{m+1,0}\left(y_{1}, \ldots, y_{m+1}\right)$ is

$$
x_{1}^{2^{m}-1} x_{2}^{2\left(2^{m}-1\right)} \ldots x_{m}^{2^{m-1}\left(2^{m}-1\right)} x_{m+1}^{2^{m}\left(2^{m}-1\right)} .
$$

Hence, by Lemma 6.2,

$$
Q_{m+1,0}\left(y_{1}, \ldots, y_{m+1}\right)=Q_{m+1,0}^{2^{m}-1}+\text { (others). }
$$

We see that if $Q_{m+1,0}^{i_{0}} Q_{m+1,1}^{i_{1}} \ldots Q_{m+1, m}^{i_{m}}$ is a term of $Q_{m+1,0}\left(y_{1}, \ldots, y_{m+1}\right)$ then

$$
i_{0}\left(2^{m+1}-1\right)+i_{1}\left(2^{m+1}-2\right)+\cdots+i_{m}\left(2^{m+1}-2^{m}\right)=\left(2^{m+1}-1\right)\left(2^{m}-1\right) .
$$

Since $2^{i} \geq 1$ for $i=0, \ldots, m$, we get

$$
\left(2^{m+1}-1\right)\left(2^{m}-1\right) \leq\left(2^{m+1}-1\right)\left(i_{0}+i_{1}+\cdots+i_{m}\right) .
$$

So

$$
2^{m}-1 \leq\left(i_{0}+i_{1}+\cdots+i_{m}\right) .
$$

On the other hand, for $j=1, \ldots, m$ we have

$$
Q_{m+1, j}=Q_{m, j-1}^{2}+\sum_{s=0}^{m}\left(Q_{m, j} Q_{m, s}\right)\left(x_{1}, \ldots, x_{m}\right) x_{m+1}^{2^{s}} \text {. }
$$

So

$Q_{m+1,0}^{i_{0}} Q_{m+1,1}^{i_{1}} \ldots Q_{m+1, m}^{i_{m}}=Q_{m, 0}^{i_{0}} \ldots Q_{m, m}^{i_{m}} x_{m+1}^{2^{m}\left(\sum_{j=0}^{m} i_{j}\right)}+$ (smaller terms). 
From the above conclusion, the powers of $x_{m+1}$ in the maximal monomial of $Q_{m+1,0}\left(y_{1}, \ldots, y_{m+1}\right)$ is $2^{m}\left(2^{m}-1\right)$. It follows that $2^{m}\left(\sum_{j=0}^{m} i_{j}\right) \leq$ $2^{m}\left(2^{m}-1\right)$. Or equivalently $\sum_{j=0}^{m} i_{j} \leq 2^{m}-1$. Combining this with (2), we get $\sum_{j=0}^{m} i_{j}=2^{m}-1$. It follows that $i_{1}=\cdots=i_{m}=0$ and $i_{0}=2^{m}-1$. Then $Q_{m+1,0}^{i_{0}} Q_{m+1,1}^{i_{1}} \cdots Q_{m+1, m}^{i_{m}}=Q_{m+1,0}^{2^{m}-1}$. As a consequence

$$
Q_{m+1,0}\left(y_{1}, \ldots, y_{m+1}\right)=Q_{m+1,0}^{2^{m}-1} .
$$

So, we get the corollary.

The following is numbered as Corollary 1.6 in the introduction.

Corollary 5.4 For $r>0, \operatorname{tr}_{2,3}\left(Q_{2,0}^{r} Q_{2,1}^{r}\right)$

$$
\begin{aligned}
= & \sum_{7 s_{0}+6 s_{1}+4 s_{2}=r} \frac{\left(s_{0}+s_{1}+s_{2}-1\right) !}{\left(s_{0}-1\right) ! s_{1} ! s_{2} !}\left(Q_{3,0}^{3} Q_{3,1} Q_{3,2}^{2}+Q_{3,0}^{5}\right)^{s_{0}}\left(Q_{3,0}^{2} Q_{3,2}^{4}\right)^{s_{1}} \\
& \times\left(Q_{3,1}^{2} Q_{3,2}^{2}+Q_{3,0}^{2} Q_{3,1}\right)^{s_{2}} .
\end{aligned}
$$

Proof Setting

$$
y_{1}=Q_{2,0} Q_{2,1}\left(x_{2}, x_{3}\right), y_{2}=Q_{2,0} Q_{2,1}\left(x_{1}, x_{3}\right), y_{3}=Q_{2,0} Q_{2,1}\left(x_{1}, x_{2}\right) .
$$

Since $Q_{2,0} Q_{2,1}$ is multilinear and alternating by Example 4.8, applying Theorem 5.2 we get

$$
\operatorname{tr}_{2,3}\left(Q_{2,0}^{r} Q_{2,1}^{r}\right)=\sum_{7 s_{0}+6 s_{1}+4 s_{2}=r} \frac{\left(s_{0}+s_{1}+s_{2}-1\right) !}{\left(s_{0}-1\right) ! s_{1} ! s_{2} !}\left(Q_{3,0}^{s_{0}} Q_{3,1}^{s_{1}} Q_{3,2}^{s_{2}}\right)\left(y_{1}, y_{2}, y_{3}\right)
$$

Now, applying Lemma 5.5, we get the corollary.

The following technical lemma will be proved in the next section.

Lemma 5.5 Let $y_{1}=Q_{2,0} Q_{2,1}\left(x_{2}, x_{3}\right), y_{2}=Q_{2,0} Q_{2,1}\left(x_{1}, x_{3}\right), y_{3}=Q_{2,0} Q_{2,1}$ $\left(x_{1}, x_{2}\right)$. Then

$$
\begin{aligned}
& Q_{3,0}\left(y_{1}, y_{2}, y_{3}\right)=Q_{3,0}^{3} Q_{3,1} Q_{3,2}^{2}+Q_{3,0}^{5}, \\
& Q_{3,1}\left(y_{1}, y_{2}, y_{3}\right)=Q_{3,0}^{2} Q_{3,2}^{4}, \\
& Q_{3,2}\left(y_{1}, y_{2}, y_{3}\right)=Q_{3,1}^{2} Q_{3,2}^{2}+Q_{3,0}^{2} Q_{3,1} .
\end{aligned}
$$

Next, we study the image of transfer on multilinear and alternating invariants in general. The following is also numbered as Proposition 1.7(i) in the introduction.

Proposition 5.6 If $Q \in D_{m}$ is multilinear and alternating then $\operatorname{tr}_{m, n}(Q)=0$. 
Proof From Proposition 4.5, it is sufficient to prove $t r_{m, n} \omega_{\beta}=0$ with $\beta=$ $\left(s_{0}, s_{1}, \ldots, s_{m-1}\right)$ such that $s_{i} \neq s_{j}$ for all $i \neq j$.

By the definition of $t r_{m, n}$ we have

$$
t r_{m, n} \omega_{\beta}=\sum_{W \leq \mathbb{V}^{*}, \operatorname{dim} W=m} \omega_{\beta}(W) .
$$

Since $\omega_{\beta}$ is multilinear and alternating, for each $m$-dimensional subspace $W$ of $\mathbb{V}^{*}$, there exist scalars $c_{i_{1}, \ldots, i_{m}}$ such that

$$
\omega_{\beta}(W)=\sum_{1 \leq i_{1}<\ldots<i_{m} \leq n} c_{i_{1}, \ldots, i_{m}} \omega_{\beta}\left(x_{i_{1}}, \ldots, x_{i_{m}}\right) .
$$

So

$$
t r_{m, n} \omega_{\beta}=\sum_{1 \leq i_{1}<\ldots<i_{m} \leq n} d_{i_{1}, \ldots, i_{m}} \omega_{\beta}\left(x_{i_{1}}, \ldots, x_{i_{m}}\right)
$$

for some scalars $d_{i_{1}, \ldots, i_{m}} \in \mathbb{F}_{2}$. From the definition of $\omega_{\beta}$ given in Sect. 4, for each sequence $\left(i_{1}, \ldots, i_{m}\right)$ we have

$$
\omega_{\beta}\left(x_{i_{1}}, \ldots, x_{i_{m}}\right)=x_{i_{1}}^{2^{s_{1}}} \ldots x_{i_{m}}^{2^{s_{m}}}+(\text { symmetried })
$$

We see that the set $\left\{x_{i_{1}}^{2^{s_{1}}} \ldots x_{i_{m}}^{2^{s_{m}}} \mid 1 \leq i_{1}<\cdots<i_{m} \leq n\right\}$ is linearly independent in the vector space of polynomials in variables $x_{1}, \ldots, x_{n}$. So, the set $\left\{\omega_{\beta}\left(x_{i_{1}}, \ldots, x_{i_{m}}\right) \mid 1 \leq i_{1}<\cdots<i_{m} \leq n\right\}$ is also linearly independent in this vector space. Furthermore, each element of this set is obtained by another one under a permutation on $\{1, \ldots, n\}$. On the other hand, as $t r_{m, n} \omega_{\beta} \in D_{n}, t r_{m, n} \omega_{\beta}$ is invariant under all permutations on $\{1, \ldots, n\}$. It implies that all coefficients $d_{i_{1}, \ldots, i_{m}}$ are equal. We denote these coefficents by $d$ for short. Then, we get

$$
t r_{m, n} \omega_{\beta}=d\left(\sum_{1 \leq i_{1}<\ldots<i_{m} \leq n} \omega_{\beta}\left(x_{i_{1}}, \ldots, x_{i_{m}}\right)\right) .
$$

Let $\sigma$ be the linear transformation which sends $x_{1}$ to $x_{1}+x_{2}$ and keeps the other variables fixed. As $\omega_{\beta}$ is multilinear and alternating, we obtain $\sigma \omega_{\beta}\left(x_{1}, x_{2}, x_{i_{3}}, \ldots, x_{i_{m}}\right)=\omega_{\beta}\left(x_{1}+x_{2}, x_{2}, x_{i_{3}}, \ldots, x_{i_{m}}\right)=\omega_{\beta}\left(x_{1}, x_{2}, x_{i_{3}}, \ldots, x_{i_{m}}\right)$

for all sequences $\left(i_{3}, \ldots, i_{m}\right)$ such that $3 \leq i_{3}<\cdots<i_{m} \leq n$;

$$
\begin{aligned}
\sigma \omega_{\beta}\left(x_{1}, x_{i_{2}}, \ldots, x_{i_{m}}\right) & =\omega_{\beta}\left(x_{1}+x_{2}, x_{i_{2}}, \ldots, x_{i_{m}}\right) \\
& =\omega_{\beta}\left(x_{1}, x_{i_{2}}, \ldots, x_{i_{m}}\right)+\omega_{\beta}\left(x_{2}, x_{i_{2}}, \ldots, x_{i_{m}}\right)
\end{aligned}
$$


for all sequences $\left(i_{2}, \ldots, i_{m}\right)$ such that $3 \leq i_{2}<\cdots<i_{m} \leq n$; and,

$$
\sigma \omega_{\beta}\left(x_{i_{1}}, \ldots, x_{i_{m}}\right)=\omega_{\beta}\left(x_{i_{1}}, \ldots, x_{i_{m}}\right)
$$

for all sequences $\left(i_{1}, \ldots, i_{m}\right)$ such that $1 \leq i_{1}<\cdots<i_{m} \leq n$. Therefore

$$
\sigma t r_{m, n} \omega_{\beta}=t r_{m, n} \omega_{\beta}+d \sum_{3 \leq i_{2}<\ldots<i_{m} \leq n} \omega_{\beta}\left(x_{2}, x_{i_{2}}, \ldots, x_{i_{m}}\right) .
$$

On the other hand $\sigma t r_{m, n} \omega_{\beta}=t r_{m, n} \omega_{\beta}$, it implies that

$$
d\left(\sum_{3 \leq i_{2}<\ldots<i_{m} \leq n} \omega_{\beta}\left(x_{2}, x_{i_{2}}, \ldots, x_{i_{m}}\right)\right)=0 .
$$

However $\sum_{3 \leq i_{2}<\cdots<i_{m} \leq n} \omega_{\beta}\left(x_{2}, x_{i_{2}}, \ldots, x_{i_{m}}\right) \neq 0$ as $n \geq m+1$. So, we get $d=0$. As a consequence $t r_{m, n} \omega_{\beta}=0$. Thus, the proposition is proved.

\section{Proof of Lemma 5.5}

In this section, we prove Lemma 5.5. However, this method can be used for finding momomials in the image of $t r_{m, n}$ on a given invariant of $D_{m}$, the proof of Corollary 5.3(ii) for an example.

Recall that $x_{1}, \ldots, x_{n}$ is a basis of $\mathbb{V}^{*}$ and for an invariant $Q \in D_{m}$,

$$
t r_{m, n}(Q)=\sum_{W \leq \mathbb{V}^{*}, \operatorname{dim} W=m} Q(W) .
$$

Definition 6.1 In $\mathbb{F}_{2}\left[x_{1}, \ldots, x_{n}\right]$, we say that $x_{1}^{i_{1}} x_{2}^{i_{2}} \ldots x_{n}^{i_{n}}>x_{1}^{j_{1}} x_{2}^{j_{2}} \ldots x_{n}^{j_{n}}$ if $\left(i_{1}, i_{2}, \ldots, i_{n}\right)>\left(j_{1}, j_{2}, \ldots, j_{n}\right)$. Here the order on the set of sequences of nonnegative integers is the colexicographical one.

For $I=\left(i_{0}, \ldots, i_{s-1}\right)$ and $W$ be an $m$-dimensional subspace of $\mathbb{V}^{*}$, we denote by $Q^{I}(W)$ the polynomial $\left(Q_{m, 0}^{i_{0}} \ldots Q_{m, m-1}^{i_{m-1}}\right)(W)$. It should be noted that $Q^{I}(W)$ is a polynomial in $\mathbb{F}_{2}\left[x_{1}, \ldots, x_{n}\right]$. Denote by $x^{I}(W)$ the maximal monomial of $Q^{I}(W)$ (with respect to the order defined in Definition 6.1).

Lemma 6.2 $x^{I}\left(\mathbb{V}^{*}\right)=x_{1}^{i_{0}} x_{2}^{2\left(i_{0}+i_{1}\right)} \ldots x_{n-1}^{2^{n-2}\left(i_{0}+i_{1}+\cdots+i_{n-2}\right)} x_{n}^{2^{n-1}\left(i_{0}+i_{1}+\cdots+i_{n-1}\right)}$ and $x^{I}\left(\mathbb{V}^{*}\right)=x^{J}\left(\mathbb{V}^{*}\right)$ if and only if $Q^{I}\left(\mathbb{V}^{*}\right)=Q^{J}\left(\mathbb{V}^{*}\right)$.

Proof The lemma is proved by induction on $n$. We use $x^{I_{n}}\left(x_{1}, \ldots, x_{n}\right)$ to stand for $x^{I}\left(\mathbb{V}^{*}\right)$.

The conclusion is trivial for $n=1$, as $Q^{I}\left(\mathbb{V}^{*}\right)=Q_{1,0}^{i_{0}}=x_{1}^{i_{0}}$. Suppose inductively that the lemma holds for $n-1$. Then we have

$$
x^{I_{n-1}}\left(x_{1}, \ldots, x_{n-1}\right)=x_{1}^{i_{0}} x_{2}^{2 .\left(i_{0}+i_{1}\right)} \ldots x_{n-1}^{2^{n-2}\left(i_{0}+i_{1}+\cdots+i_{n-2}\right)} .
$$


Using the inductive formula $Q_{n, 0}=Q_{n-1,0} V_{n}, Q_{n, i}=Q_{n-1, i-1}^{2}+Q_{n-1, i} V_{n}$ $(1 \leq i \leq n-1)$ for $i \leq 1 \leq n-1$ and $V_{n}\left(x_{1}, \ldots, x_{n-1}, x_{n}\right)=\sum_{j=0}^{n-1} Q_{n-1, j} x_{n}^{2^{j}}$, we get

$$
\begin{aligned}
Q_{n, 0} & =\sum_{j=0}^{n-1}\left(Q_{n-1,0} Q_{n-1, j}\right) x_{n}^{2^{j}} . \\
Q_{n-1, i} & =Q_{n-1, i-1}^{2}+\sum_{j=0}^{n-1}\left(Q_{n-1, i} Q_{n-1, j}\right) x_{n}^{2^{j}} \quad(1 \leq i \leq n-1) .
\end{aligned}
$$

Therefore

$$
\begin{aligned}
Q^{I}\left(\mathbb{V}^{*}\right)= & \left(Q_{n-1,0}^{i_{0}} \ldots Q_{n-1, n-2}^{i_{n-2}}\right) x_{n}^{2^{n-1}\left(i_{0}+i_{1}+\ldots+i_{n-1}\right)} \\
& +\left(\text { other monomials in which the powers of } x_{n} \text { are smaller }\right) \\
= & x^{I_{n-1}}\left(x_{1}, \ldots, x_{n-1}\right) x_{n}^{2^{n-1}\left(i_{0}+i_{1}+\ldots+i_{n-1}\right)}+(\text { smaller monomials }) \\
= & x_{1}^{i_{0}} x_{2}^{2\left(i_{0}+i_{1}\right)} \ldots x_{n-1}^{2^{n-2}\left(i_{0}+i_{1}+\cdots+i_{n-2}\right)} x_{n}^{2^{n-1}\left(i_{0}+i_{1}+\cdots+i_{n-1}\right)} \\
& +(\text { smaller monomials })
\end{aligned}
$$

as $x^{I_{n-1}}\left(x_{1}, \ldots, x_{n-1}\right)=x_{1}^{i_{0}} x_{2}^{2\left(i_{0}+i_{1}\right)} \ldots x_{n-1}^{2^{n-2}\left(i_{0}+i_{1}+\cdots+i_{n-2}\right)}$ by the inductive hypothesis. So

$$
x^{I_{n}}\left(x_{1}, \ldots, x_{n}\right)=x_{1}^{i_{0}} x_{2}^{2\left(i_{0}+i_{1}\right)} \ldots x_{n-1}^{2^{n-2}\left(i_{0}+i_{1}+\cdots+i_{n-2}\right)} x_{n}^{2^{n-1}\left(i_{0}+i_{1}+\cdots+i_{n-1}\right)} .
$$

Then we get the first part of the lemma.

Suppose that $I=\left(i_{0}, \ldots, i_{n}\right)$ and $J=\left(j_{0}, \ldots, j_{n}\right)$. We see that $x^{I}\left(\mathbb{V}^{*}\right)=$ $x^{J}\left(\mathbb{V}^{*}\right)$ if and only if

$$
\left\{\begin{array} { l } 
{ i _ { 0 } = j _ { 0 } } \\
{ 2 ( i _ { 0 } + i _ { 1 } ) = 2 ( j _ { 0 } + j _ { 1 } ) } \\
{ \cdots } \\
{ 2 ^ { n - 1 } ( i _ { 0 } + i _ { 1 } + \cdots + i _ { n - 1 } ) = 2 ^ { n - 1 } ( j _ { 0 } + j _ { 1 } + \cdots + j _ { n - 1 } ) }
\end{array} \Longleftrightarrow \left\{\begin{array}{l}
i_{0}=j_{0} \\
i_{1}=j_{1} \\
\ldots \\
i_{n}=j_{n} .
\end{array}\right.\right.
$$

So, we obtain the remaining part of the lemma.

Proof of Lemma 5.5 By direct computation the Dickson invariants $Q_{2,0}, Q_{2,1}$ in terms of variables, we get

$$
\begin{aligned}
& y_{1}=Q_{2,0} Q_{2,1}\left(x_{2}, x_{3}\right)=x_{2} x_{3}^{4}+x_{2}^{4} x_{3}, \\
& y_{2}=Q_{2,0} Q_{2,1}\left(x_{1}, x_{3}\right)=x_{1} x_{3}^{4}+x_{1}^{4} x_{3}, \\
& y_{3}=Q_{2,0} Q_{2,1}\left(x_{1}, x_{2}\right)=x_{1} x_{2}^{4}+x_{1}^{4} x_{2} .
\end{aligned}
$$


By the definition of $Q_{3,0}$, we get

$$
Q_{3,0}\left(y_{1}, y_{2}, y_{3}\right)=y_{1}^{4} y_{2}^{2} y_{3}+\left(\text { symmetried terms in } y_{1}, y_{2}, y_{3}\right) \text {. }
$$

And, by the definition of $y_{1}, y_{2}, y_{3}$, we have

$$
\begin{aligned}
y_{1}^{4} y_{2}^{2} y_{3}= & \left(x_{1}^{3} x_{2}^{8}+x_{1}^{6} x_{2}^{5}\right) x_{3}^{24}+\left(x_{1}^{9} x_{2}^{8}+x_{1}^{12} x_{2}^{5}\right) x_{3}^{18}+\left(x_{1}^{3} x_{2}^{20}+x_{1}^{6} x_{2}^{17}\right) x_{3}^{12} \\
& +\left(x_{1}^{9} x_{2}^{10}+x_{1}^{12} x_{2}^{17}\right) x_{3}^{6} .
\end{aligned}
$$

We see that if $\sigma$ is a permutation on the set $\{1,2,3\}$, then $x_{1}^{a} x_{2}^{b} x_{3}^{c}$ is a term of $y_{1}^{4} y_{2}^{2} y_{3}$ iff $x_{\sigma(1)}^{a} x_{\sigma(2)}^{b} x_{\sigma(3)}^{c}$ is a term of the symmetried one $y_{\sigma(1)} y_{\sigma(2)}^{2} y_{\sigma(3)}^{4}$. So, as $x_{1}^{3} x_{2}^{8} x_{3}^{24}$ is the maximal monomial of $y_{1} y_{2}^{2} y_{3}^{4}$, it is also the maximal monomial of $Q_{3,0}\left(y_{1}, y_{2}, y_{3}\right)$. Therefore, by Lemma 6.2, $Q_{3,0}\left(y_{1}, y_{2}, y_{3}\right)$ contains $Q_{3,0}^{i_{0}} Q_{3,1}^{i_{1}} Q_{3,2}^{i_{2}}$ as a term in which

$$
\left\{\begin{array} { l } 
{ 4 ( i _ { 0 } + i _ { 1 } + i _ { 2 } ) = 2 4 } \\
{ 2 ( i _ { 0 } + i _ { 1 } ) = 8 } \\
{ i _ { 0 } = 3 }
\end{array} \Longleftrightarrow \left\{\begin{array}{l}
i_{0}=3 \\
i_{1}=1 \\
i_{2}=2
\end{array}\right.\right.
$$

So

$$
Q_{3,0}\left(y_{1}, y_{2}, y_{3}\right)=Q_{3,0}^{3} Q_{3,1} Q_{3,2}^{2}+\text { (other terms). }
$$

We get

$$
\begin{aligned}
Q_{3,0}^{3} Q_{3,1} Q_{3,2}^{2}= & \left(Q_{3,0} Q_{3,1}\right)\left(Q_{3,0} Q_{3,2}\right)^{2} \\
= & \left(x_{1} x_{2}^{4} x_{3}^{8}+(\text { symmetried })\right)\left(x_{1} x_{2}^{2} x_{3}^{8}+(\text { symmetried })\right)^{2} \\
= & \left(x_{1} x_{2}^{4} x_{3}^{8}+(\text { symmetried })\right)\left(x_{1}^{2} x_{2}^{4} x_{3}^{16}+\text { (symmetried) }\right) \\
= & \left(x_{1}^{4} x_{2}^{8}+x_{1}^{6} x_{2}^{7}\right) x_{3}^{24}+\left(x_{1}^{6} x_{2}^{12}+x_{1}^{5} x_{2}^{10}\right) x_{3}^{20} \\
& +\left(x_{1}^{6} x_{2}^{12}+x_{1}^{8} x_{2}^{10}\right) x_{3}^{17}+\text { (symmetried). }
\end{aligned}
$$

Therefore

$$
\begin{aligned}
Q_{3,0}\left(y_{1}, y_{2}, y_{3}\right)+Q_{3,0}^{3} Q_{3,1} Q_{3,2}^{2}= & x_{1}^{5} x_{2}^{10} x_{3}^{20}+x_{1}^{5} x_{2}^{12} x_{3}^{18}+x_{1}^{8} x_{2}^{9} x_{3}^{18} \\
& +x_{1}^{6} x_{2}^{12} x_{3}^{17}+x_{1}^{8} x_{2}^{10} x_{3}^{17}+(\text { symmetried }) .
\end{aligned}
$$

The right hand side is an invariant whose the maximal monomial is $x_{1}^{5} x_{2}^{10} x_{3}^{20}$. So, by Lemma 6.2 again, it contains $Q_{3,0}^{3}$ as a term. Furthermore, by direct computation, it is equal to $Q_{3,0}^{3}$. Hence 


$$
Q_{3,0}\left(y_{1}, y_{2}, y_{3}\right)=Q_{3,0}^{3} Q_{3,1} Q_{3,2}^{2}+Q_{3,0}^{3}
$$

Similarly as in the proof of the first part, we have

$$
\begin{aligned}
Q_{3,1}\left(y_{1}, y_{2}, y_{3}\right)= & y_{1}^{4} y_{2}^{2}+y_{1}^{4} y_{2} y_{3}+y_{1}^{2} y_{2}^{2} y_{3}^{2}+\text { (symmetried) } \\
= & x_{1}^{2} x_{2}^{4} x_{3}^{24}+x_{1}^{2} x_{2}^{8} x_{3}^{20}+x_{1}^{4} x_{2}^{8} x_{3}^{18} \\
& +\left(x_{1}^{2} x_{2}^{12}+x_{1}^{4} x_{2}^{10}+x_{1}^{6} x_{2}^{8}\right) x_{3}^{16}+\text { (symmetried). }
\end{aligned}
$$

The right hand side is an invariant whose the maximal monomial is $x_{1}^{2} x_{2}^{4} x_{3}^{24}$. So, by Lemma 6.2, it contains $Q_{3,0}^{2} Q_{3,2}^{4}$ as a term. Furthermore it is equal to $Q_{3,0}^{2} Q_{3,2}^{4}$; so,

$$
Q_{3,1}\left(y_{1}, y_{2}, y_{3}\right)=Q_{3,0}^{2} Q_{3,2}^{4} \text {. }
$$

So we get the second part of the lemma. Similarly, we have

$$
\begin{aligned}
Q_{3,2}\left(y_{1}, y_{2}, y_{3}\right)= & y_{1}^{4}+y_{1}^{2} y_{2} y_{3}+y_{1}^{2} y_{2}^{2}+(\text { symmetried }) \\
= & x_{2}^{4} x_{3}^{16}+x_{1}^{2} x_{2}^{2} x_{3}^{16}+x_{1}^{2} x_{2}^{8} x_{3}^{10}+\left(x_{1}^{2} x_{2}^{6}+x_{1}^{3} x_{2}^{5}\right) x_{3}^{12} \\
& +\left(x_{1}^{5} x_{2}^{6}+x_{1}^{3} x_{2}^{8}\right) x_{3}^{9}+x_{1}^{4} x_{2}^{8} x_{3}^{8}+\text { (symmetried). }
\end{aligned}
$$

The right hand side is an invariant whose the maximal monomial is $x_{2}^{2} x_{3}^{16}$. So, by Lemma 6.2, it contains $Q_{3,1}^{2} Q_{3,2}^{2}$ as a term. We see that

$$
\begin{aligned}
Q_{3,2}\left(y_{1}, y_{2}, y_{3}\right)+Q_{3,1}^{2} Q_{3,2}^{2}= & \left(x_{1}^{2} x_{2}^{6}+x_{1}^{3} x_{2}^{5}\right) x_{3}^{12}+\left(x_{1}^{3} x_{2}^{8}+x_{1}^{5} x_{2}^{6}\right) x_{3}^{9} \\
& +(\text { symmetried }) .
\end{aligned}
$$

The maximal monomial of this invariant is $x_{1}^{2} x_{2}^{6} x_{3}^{12}$. So, by Lemma 6.2, it contains $Q_{3,0}^{2} Q_{3,1}$ as a term. Furthermore, by direct computation, we obtain

$$
Q_{3,2}\left(y_{1}, y_{2}, y_{3}\right)+Q_{3,1}^{2} Q_{3,2}^{2}=Q_{3,0}^{2} Q_{3,1} \text {. }
$$

So

$$
Q_{3,2}\left(y_{1}, y_{2}, y_{3}\right)=Q_{3,1}^{2} Q_{3,2}^{2}+Q_{3,0}^{2} Q_{3,1}
$$

Hence, the lemma is completely proved.

\section{Some invariants on which the transfer vanishes}

The goal of this section is to prove the two following propositions, which are numbered as Proposition 1.7(ii) and (iii) respectively. 
Proposition 7.1 $\operatorname{tr}_{m, n}\left(Q_{m, 0}^{2^{s_{0}}} Q_{m, 1}^{2^{s_{1}}} \ldots Q_{m, m-1}^{2^{s_{m-1}}}\right)=0$ for any nonnegative integers $s_{0}, s_{1}, \ldots, s_{m-1}$ and positive integers $m, n$ such that $n \geq \frac{3 m^{2}+3 m}{2}$.

Proof Setting $s=\min \left\{s_{0}, s_{1}, \ldots, s_{m-1}\right\}$ and suppose that $s=s_{i}$ for some $0 \leq i \leq$ $m-1$. For $0 \leq j \leq m-1$, let $t_{j}=s_{j}-s$. So, we have $t_{i}=0$ and

$$
t r_{m, n}\left(Q_{m, 0}^{2^{s_{0}}} Q_{m, 1}^{2^{s_{1}}} \ldots Q_{m, m-1}^{2^{s_{m-1}}}\right)=\left(t r_{m, n}\left(Q_{m, 0}^{2^{t_{0}}} Q_{m, 1}^{2^{t_{1}}} \ldots Q_{m, m-1}^{2^{t_{m-1}}}\right)\right)^{2^{s}}
$$

Denote $Q=t r_{m, n}\left(Q_{m, 0}^{2^{t_{0}}} Q_{m, 1}^{2^{t_{1}}} \ldots Q_{m, m-1}^{2^{t_{m-1}}}\right)$. We will show that $Q=0$. Suppose to the contrary that $Q \neq 0$, by the definition of $t r_{m, n}$, we get

$$
Q=\sum Q_{n, 0}^{i_{0}} Q_{n, 1}^{i_{1}} \ldots Q_{n, n-1}^{i_{n-1}}
$$

for some sequences of nonnegative integers $\left(i_{1}, i_{1}, \ldots, i_{n-1}\right)$. We consider the maximal monomial in variables $x_{1}, \ldots, x_{n}$ of $Q$, denoted by $x(Q)$.

First, from Lemma 6.2, there is exactly one invariant monomial $Q_{n, 0}^{i_{0}} Q_{n, 1}^{i_{1}} \ldots$ $Q_{n, n-1}^{i_{n-1}}$ of $Q$ containing $x(Q)$; furthermore,

$$
x(Q)=x_{1}^{i_{0}} x_{2}^{2\left(i_{0}+i_{1}\right)} \ldots x_{n}^{2^{n-1}\left(i_{0}+i_{1}+\cdots+i_{n-1}\right)} .
$$

Let $h$ be the maximal index such that $i_{h} \neq 0$. Then $i_{0}=\cdots=i_{h-1}=0$. So

$$
\begin{aligned}
x(Q)= & x_{h+1}^{2^{h} \cdot i_{h}} x_{h+2}^{2^{h+1}\left(i_{h}+i_{h+1}\right)} \ldots x_{n}^{2^{n-1}\left(i_{h} \cdots+i_{n-1}\right)}, \\
& \Longrightarrow \operatorname{deg}(x(Q))=2^{h} \cdot i_{h}+2^{h+1}\left(i_{h}+i_{h+1}\right)+\cdots+2^{n-1}\left(i_{h}+\cdots+i_{n-1}\right) .
\end{aligned}
$$

It implies that $2^{h}$ divides $\operatorname{deg}(x(Q))$. On the other hand $\operatorname{deg}(x(Q))=\sum_{j=0}^{m}\left(2^{m}-\right.$ $\left.2^{j}\right) 2^{t_{j}}$; so, $2^{h}$ divides $\sum_{j=0}^{m}\left(2^{m}-2^{j}\right) 2^{t_{j}}$.

Second, we see that each invariant $Q_{m, j}$ with $0 \leq j \leq m-1$ is a polynomial in $m$ variables. Thus, if $W$ is an $m$-dimensional subspace of $\mathbb{V}^{*}$ then $\left(Q_{m, 0}^{2^{t_{0}}} Q_{m, 1}^{2^{t_{1}}} \ldots Q_{m, m-1}^{2^{t_{m-1}}}\right)(W)$ is a polynomial of at most $m^{2}$ distinct variables. Thus, by the definition of $t r_{m, n}, Q=\sum_{W \leq \mathbb{V}^{*}, \operatorname{dim} W=m}\left(Q_{m, 0}^{2^{t_{0}}} Q_{m, 1}^{2^{t_{1}}} \ldots Q_{m, m-1}^{2^{t_{m-1}}}\right)(W)$ is a polynomial of at most $m^{2}$ distinct variables. As $x(Q)$ is a monomial of $Q$, from the formula of $x(Q)$ given above, we get the number of distinct variables of $x(Q), n-h$, is less than or equal to $m^{2}$. Combining with the hypothesis $n \geq \frac{3 m^{2}+3 m}{2}$, we obtain

$$
h \geq n-m^{2} \geq \frac{3 m^{2}+3 m}{2}-m^{2}=\frac{m^{2}+3 m}{2} .
$$

So $2^{\frac{m^{2}+3 m}{2}}$ divides $2^{h}$. As a consequence $2^{\frac{m^{2}+3 m}{2}}$ divides $\sum_{j=0}^{m}\left(2^{m}-2^{j}\right) 2^{t_{j}}$. Let $2^{\alpha_{1}}+\cdots+2^{\alpha_{k}}$ be the dyadic expansion of $\sum_{j=0}^{m}\left(2^{m}-2^{j}\right) 2^{t_{j}}$ with $\alpha_{1}<\cdots<\alpha_{k}$. It implies that

$$
\alpha_{i}>2^{\frac{m^{2}+3 m}{2}} \text { for all } i \geq 1 \text {. }
$$


Third, since $t_{i}=0$ we get

$$
\begin{aligned}
\sum_{j=0}^{m}\left(2^{m}-2^{j}\right) 2^{t_{j}}= & \left(2^{m-1}+\cdots+1\right) 2^{t_{1}}+\cdots+\left(2^{m-1}+\cdots+2^{i}\right) \\
& +\cdots+2^{m-1} \cdot 2^{t_{m-1}} .
\end{aligned}
$$

Now, we reduce this sum to the dyadic expansion form $2^{\alpha_{1}}+\cdots+2^{\alpha_{k}}$ given above. If $2^{i}$ is a term in the dyadic expansion form, then there exists an index $j$ such that $i=\alpha_{j}$. As $i \leq m-1$, we get

$$
\alpha_{j}<m<\frac{m^{2}+3 m}{2} \text {. }
$$

This contradicts the inequality (3). If $2^{i}$ does not appear in the the dyadic expansion form, then there exists an positive integer $k$ and an index $j$ such that

$$
2^{i}+2^{i}+2^{i+1}+\cdots+2^{i+k}=2^{i+k}=2^{\alpha_{j}} .
$$

It should be noted that the number of powers of 2 in the sum $\sum_{j=0}^{m}\left(2^{m}-2^{j}\right) 2^{t_{j}}$ is $(m-1)+(m-2)+\cdots+1=\frac{m^{2}+m}{2}$. So $k \leq \frac{m^{2}+m}{2}$. Therefore

$$
\alpha_{j}=k+i \leq \frac{m^{2}+m}{2}+i<\frac{m^{2}+m}{2}+m=\frac{m^{2}+3 m}{2}
$$

(as $i<m$ ). This contradicts the inequality (3). As a consequence, we get $Q=$ $t r_{m, n}\left(Q_{m, 0}^{2^{t_{0}}} Q_{m, 1}^{2^{t_{1}}} \ldots Q_{m, m-1}^{2^{t_{m-1}}}\right)=0$. So

$$
t r_{m, n}\left(Q_{m, 0}^{2^{s_{0}}} Q_{m, 1}^{2^{s_{1}}} \cdots Q_{m, m-1}^{2^{s_{m-1}}}\right)=0
$$

Hence, the proposition is completely proved.

For case $m=2$, we get the following.

Proposition 7.2 $\operatorname{tr}_{2, n}\left(Q_{2,0}^{2^{s_{0}}} Q_{2,1}^{2^{s_{1}}}\right)=0$ for any nonnegative intergers $s_{0}, s_{1}$ and $n \geq 4$.

Remark 7.3 The statement in Proposition 7.2 is no longer true for $n=3$. In fact, we have

(i) if $s_{0}-s_{1}>1$ then $\operatorname{tr}_{2,3}\left(Q_{2,0}^{2^{s_{0}}} Q_{2,1}^{2^{s_{1}}}\right)=Q_{3,0}^{2^{s_{1}}} Q_{3,1}^{2^{s_{0}-1}-2^{s_{1}}}+$ (others),

(ii) if $s_{0}-s_{1}=0$ or 1 then $\operatorname{tr}_{2,3}\left(Q_{2,0}^{2^{s_{0}}} Q_{2,1}^{2^{s_{1}}}\right)=0$,

(iii) if $s_{0}-s_{1}<0$ then $\operatorname{tr}_{2,3}\left(Q_{2,0}^{2^{s_{0}}} Q_{2,1}^{2^{s_{1}}}\right)=Q_{3,0}^{2^{s_{0}}} Q_{3,2}^{2^{s_{1}-1}-2^{s_{0}}}+$ (others). 
Proof Case 1. $s_{0}>s_{1}$. We have $t r_{2,3}\left(Q_{2,0}^{2^{s_{0}}} Q_{2,1}^{2^{s_{1}}}\right)=\left(\operatorname{tr}_{2,3}\left(Q_{2,0}^{2^{s_{0}-s_{1}}} Q_{2,1}\right)\right)^{2^{s_{1}}}$. Setting $a=s_{0}-s_{1}$. By the definition of $t r_{2,3}$, we get

$$
\begin{aligned}
\operatorname{tr}_{2,3}\left(Q_{2,0}^{2^{s_{0}-s_{1}}} Q_{2,1}\right)= & \operatorname{tr}_{2,3}\left(Q_{2,0}^{2^{a}} Q_{2,1}\right) \\
= & \left(Q_{2,0}^{2^{a}} Q_{2,1}\right)\left(x_{1}, x_{2}\right)+\left(Q_{2,0}^{2^{a}} Q_{2,1}\right)\left(x_{1}, x_{3}\right) \\
& +\left(Q_{2,0}^{2^{a}} Q_{2,1}\right)\left(x_{1}, x_{3}\right)+\left(Q_{2,0}^{2^{a}} Q_{2,1}\right)\left(x_{1}+x_{2}, x_{3}\right) \\
& +\left(Q_{2,0}^{2^{a}} Q_{2,1}\right)\left(x_{1}, x_{2}+x_{3}\right)+\left(Q_{2,0}^{2^{a}} Q_{2,1}\right)\left(x_{1}+x_{3}, x_{2}+x_{3}\right) .
\end{aligned}
$$

By direct computation, we have

$$
\begin{aligned}
& \left(Q_{2,0}^{2^{a}} Q_{2,1}\right)\left(x_{1}, x_{2}\right)=x_{1}^{2^{a}} x_{2}^{2^{a+1}+2}+x_{1}^{2^{a+1}+1} x_{2}^{2^{a}+1}+x_{1}^{2^{a+1}} x_{2}^{2^{a}+2} \\
& + \text { (symmetried), } \\
& \left(Q_{2,0}^{2^{a}} Q_{2,1}\right)\left(x_{1}+x_{2}, x_{3}\right)=x_{2}^{2^{a}} x_{3}^{2^{a+1}+2}+x_{2}^{2^{a}+1} x_{3}^{2^{a+1}+1}+x_{2}^{2^{a}+2} x_{3}^{2^{a+1}}+x_{1}^{2^{a}} x_{3}^{2^{a+1}+2} \\
& +x_{1}^{2^{a}+1} x_{3}^{2^{a+1}+1}+x_{1}^{2^{a}+2} x_{3}^{2^{a+1}}+x_{1} x_{2}^{a} x_{3}^{2^{a+1}+1} \\
& +x_{1}^{2} x_{2}^{a} x_{3}^{2^{a+1}}+x_{1} x_{2}^{a+1} x_{3}^{2^{a}+1}+x_{1}^{2} x_{2}^{a+1} x_{3}^{2^{a}} \\
& +(\text { symmetried }) \text {, } \\
& \left(Q_{2,0}^{2^{a}} Q_{2,1}\right)\left(x_{1}+x_{2}, x_{2}+x_{3}\right)=\left(x_{1}^{2} x_{2}^{2^{a}} x_{3}^{2^{a+1}}+x_{1} x_{2}^{2^{a}+1} x_{3}^{2^{a+1}}\right)+(\text { symmetried }) \\
& +\sum_{T \in S_{3}} x_{T(1)}^{2^{a+1}+2} x_{T(2)}^{2^{a}}+\sum_{T \in S_{3}} x_{T(1)}^{2^{a+1}+1} x_{T(2)}^{2^{a}+1} \\
& +\sum_{T \in S_{3}} x_{T(1)}^{2^{a+1}} x_{T(2)}^{2^{a}+2} \text {. }
\end{aligned}
$$

We see that if $a=s_{0}-s_{1}=1$ then $\operatorname{tr}_{2,3}\left(Q_{2,0}^{2^{a}} Q_{2,1}\right)=0$; otherwise, if $a>1$ then

$$
\operatorname{tr}_{2,3}\left(Q_{2,0}^{2^{a}} Q_{2,1}\right)=x_{1}^{2} x_{2}^{2^{a}} x_{3}^{2^{a+1}}+\text { (symmetried) }
$$

In this case $x_{1}^{2} x_{2}^{2^{a}} x_{3}^{2^{a+1}}$ is the maximal monomial of $\operatorname{tr}_{2,3}\left(Q_{2,0}^{2^{a}} Q_{2,1}\right)$. So, by Lemma 6.2, $\operatorname{tr}_{2,3}\left(Q_{2,0}^{2^{a}} Q_{2,1}\right)$ contains the invariant $Q_{3,0}^{i_{0}} Q_{3,1}^{i_{1}} Q_{3,2}^{i_{2}}$ as a term in which

$$
\left\{\begin{array} { l } 
{ i _ { 0 } = 2 } \\
{ 2 ( i _ { 0 } + i _ { 1 } ) = 2 ^ { a } } \\
{ 4 ( i _ { 0 } + i _ { 1 } + i _ { 2 } ) = 2 ^ { a + 1 } }
\end{array} \Longleftrightarrow \left\{\begin{array}{l}
i_{0}=2 \\
i_{1}=2^{a-1}-2 \\
i_{2}=0 .
\end{array}\right.\right.
$$


Therefore $\operatorname{tr}_{2,3}\left(Q_{2,0}^{2^{a}} Q_{2,1}\right)=Q_{3,0} Q_{3,1}^{2^{a-1}-1}+$ (others). Hence

$$
\left.\operatorname{tr}_{2,3}\left(Q_{2,0}^{2^{s_{0}}} Q_{2,1}^{2^{s_{1}}}\right)=\left(Q_{3,0} Q_{3,1}^{2^{a-1}-1}+(\text { others })\right)^{2^{s_{1}}}=Q_{3,0}^{2^{s_{1}}} Q_{3,1}^{2^{s_{0}-1}-2^{s_{1}}}+\text { (others }\right)
$$

Case 2. $s_{0}=s_{1}$. From Example 4.8, we see that $Q_{2,0} Q_{2,1}$ is multilinear and alternating. So, by Proposition 5.6, $t r_{2,3}\left(Q_{2,0} Q_{2,1}\right)=0$. Hence $t r_{2,3}\left(Q_{2,0}^{2^{s_{0}}} Q_{2,1}^{2^{s_{1}}}\right)=$ $\left(\operatorname{tr}_{2,3}\left(Q_{2,0} Q_{2,1}\right)^{2^{s_{0}}}\right)^{2^{s_{0}}}=0$.

Case 3. $s_{0}<s_{1}$. Similarly as proof of Case 1 , setting $b=2^{s_{1}}-2^{s_{0}}$ we have $Q_{2,0}^{2^{s_{0}}} Q_{2,1}^{2^{s_{1}}}=\left(Q_{2,0} Q_{2,1}^{b}\right)^{2^{s_{0}}}$. And

$$
Q_{2,0} Q_{2,1}^{b}=x_{1} x_{2}^{2} x_{3}^{2^{b+1}}+\text { (symmetried). }
$$

The maximal monomial of $Q_{2,0} Q_{2,1}^{b}$ is $x_{1} x_{2}^{2} x_{3}^{2^{b+1}}$. So, by Lemma 6.2, $Q_{2,0} Q_{2,1}^{b}$ contains $Q_{3,0} Q_{3,2}^{2^{b-1}-1}$ as a term. Therefore

$$
\begin{aligned}
\operatorname{tr}_{2,3}\left(Q_{2,0}^{2^{s_{0}}} Q_{2,1}^{2^{s_{1}}}\right) & =\left(\operatorname{tr}_{2,3}\left(Q_{2,0} Q_{2,1}^{b}\right)\right)^{2^{s_{0}}} \\
& =\left(Q_{3,0} Q_{3,2}^{2^{b-1}-1}+(\text { others })\right)^{2^{s_{0}}} \\
& =Q_{3,0}^{2^{s_{0}}} Q_{3,2}^{2^{s_{1}-1}-2^{s_{0}}}+\text { (others) }
\end{aligned}
$$

Proof of Proposition 7.2 Since $t r_{2, n}=t r_{3, n} t r_{2,3}$ by [3, Lemma 2.4], we have

$$
\operatorname{tr}_{2, n}\left(Q_{2,0}^{2^{s_{0}}} Q_{2,1}^{2^{s_{1}}}\right)=\operatorname{tr}_{3, n} \operatorname{tr}_{2,3}\left(Q_{2,0}^{2^{s_{0}}} Q_{2,1}^{2^{s_{1}}}\right)
$$

Setting $Q=\operatorname{tr}_{2,3}\left(Q_{2,0}^{2^{s_{0}}} Q_{2,1}^{2^{s_{1}}}\right)$. We need to prove that $\operatorname{tr}_{3, n}(Q)=0$.

Case 1. $s_{0}-s_{1}>1$. We have

$$
\begin{aligned}
Q= & \left(\operatorname{tr}_{2,3}\left(Q_{2,0}^{2^{s_{0}-s_{1}}} Q_{2,1}\right)\right)^{2^{s_{1}}} \\
= & \left(x_{1}^{2} x_{2}^{2^{s_{0}-s_{1}}} x_{3}^{2^{s_{0}-s_{1}+1}}+(\text { symmetried })\right)^{2^{s_{1}}} \\
& \quad[\text { as shown in the proof of Remark } 7.3(\mathrm{i})] \\
= & x_{1}^{2^{s_{1}+1}} x_{2}^{2^{s_{0}}} x_{3}^{2^{s_{0}+1}}+(\text { symmetried }) .
\end{aligned}
$$

From Proposition 4.5, we see that $Q$ is the multilinear and alternating invariant $\omega_{\beta}$ with $\beta=\left(s_{1}+1, s_{0}, s_{0}+1\right)$. So, by Proposition 5.6, we get $\operatorname{tr}_{3, n}(Q)=0$.

Case 2. $s_{0}-s_{1}=1$ or 0 . Following Remark 7.3(ii), we get $Q=0$. Thus $\operatorname{tr}_{3, n}(Q)=0$. 
Case 3. $s_{0}-s_{1}<0$. We have

$$
\begin{aligned}
Q= & \left(\operatorname{tr}_{2,3}\left(Q_{2,0} Q_{2,1}^{2^{s_{1}-s_{0}}}\right)\right)^{2^{s_{0}}} \\
= & \left(x_{1} x_{2}^{2} x_{3}^{2^{s_{1}-s_{0}+1}}+(\text { symmetried })\right)^{2^{s_{0}}} \\
& {[\text { as shown in the proof of Remark 7.3(ii)] }} \\
= & x_{1}^{2^{s_{0}}} x_{2}^{2^{s_{0}}+1} x_{3}^{2^{s_{1}+1}}+(\text { symmetried) } .
\end{aligned}
$$

Similarly, from Proposition 4.5, we obtain that $Q$ is the multilinear and alternating invariant $\omega_{\beta}$ with $\beta=\left(s_{0}, s_{0}+1, s_{1}+1\right)$. So, by Proposition 5.6, we get $\operatorname{tr}_{3, n}(Q)=0$.

The proposition is proved.

Acknowledgments We would like to thank Phạm H. Đăng, who wrote an undergraduate thesis in 2011 under the guidance of Prof. Nguyễn H. V. Hưng on transfers, and is no longer studying Maths, for valuable discussion on Theorem 1.2. We also thank to Prof. Nguyễn H. V. Hưng for many helpful suggestions.

\section{References}

1. Đăng, P.H.: The transfer between the Dickson algebras. Undergraduate thesis, VNU University of Science (2011)

2. Dickson, L.E.: A fundamental system of invariants of the general modular linear group with a solution of the form problem. Trans. Amer. Math. Soc. 12, 75-98 (1911)

3. Hưng, N.H.V.: The homomorphisms between the Dickson-Mùi algebras as modules over the Steenrod algebra. Math. Ann. 353, 827-866 (2012)

4. Hưng, N.H.V., Peterson, F.P.: Spherical classes and the Dickson algebra. Math. Proc. Camb. Philos. Soc. 124, 253-264 (1998)

5. Mùi, Huỳnh: Modular invariant theory and cohomology algebras of symmetric groups. J. Fac. Sci. Univ. Tokyo Sect. IA Math. 22, 319-369 (1975) 